\title{
Influence of Ag additive to the spacer layer on the structure and giant magnetoresistance of electrodeposited $\mathrm{Co} / \mathrm{Cu}$ multilayers
}

\author{
K. Neuróhr ${ }^{1}$, L. Péter ${ }^{1}$, L. Pogány ${ }^{1}$, D. Rafaja ${ }^{2}$, A. Csik ${ }^{3}$, K. Vad ${ }^{3}$, \\ G. Molnár ${ }^{4}, \mathrm{I}$ Bakonyi ${ }^{1+}$ \\ ${ }^{1}$ Wigner Research Centre for Physics, Hungarian Academy of Sciences \\ H-1121 Budapest, Konkoly-Thege út 29-33, Hungary \\ ${ }^{2}$ Institute of Materials Science, TU Bergakademie Freiberg \\ Gustav-Zeuner-Str. 5, D-09599 Freiberg, Germany \\ ${ }^{3}$ Institute for Nuclear Research, Hungarian Academy of Sciences \\ H-4026 Debrecen, Bem tér 18/c, Hungary
}

${ }^{4}$ Institute for Materials Science and Technical Physics, Energy Research Centre,
Hungarian Academy of Sciences. H-1121 Budapest, Konkoly-Thege út 29-33, Hungary

ABSTRACT - In order to explore the possible surfactant effect of Ag on the formation of electrodeposited multilayers, $\mathrm{Co} / \mathrm{Cu}(\mathrm{Ag})$ multilayers were prepared by this technique and their structure and giant magnetoresistance (GMR) were investigated. The multilayers were deposited from a perchlorate bath with various amounts of $\mathrm{Ag}^{+}$ions in the solution for incorporating $\mathrm{Ag}$ atoms into the multilayer stack. Without Ag addition, secondary neutral mass spectroscopy (SNMS) indicated a well-defined composition modulation of the undermost $\mathrm{Co} / \mathrm{Cu}$ bilayers. However, already at an $\mathrm{Ag}$ content as low as 1.8 at.\% incorporated, SNMS showed a deterioration of the periodic multilayer structure. In agreement with the SNMS results, superlattice satellites were visible in the X-ray diffraction (XRD) patterns of the multilayers with up to 0.3 at.\% Ag. The satellites were, however, very faint even for multilayers without $\mathrm{Ag}$ addition, indicating that the multilayers have high interface roughness and/or poor periodicity. In the absence of $\mathrm{Ag}$ and at the smallest $\mathrm{Ag}$ content investigated by XRD, a strong central multilayer peak and the weak superlattice satellites were complemented by weak diffraction maxima from non-periodic $\mathrm{Co}$ and $\mathrm{Cu}$ domains. In the $\mathrm{Co} / \mathrm{Cu}(\mathrm{Ag})$ multilayer containing about 25 at.\% $\mathrm{Ag}$, i.e., nearly as much as $\mathrm{Cu}, \mathrm{XRD}$ found a separate $\operatorname{Ag}(\mathrm{Cu})$ phase. In spite of the imperfect layered structure, a multilayer-type GMR behavior was observed in all samples up to about 10 at.\% Ag incorporated in the multilayer stack. The GMR magnitude increased for Ag contents up to about 1 at.\%, which implies that a small amount of Ag may have a beneficial effect through a slight modification of the layer growth and/or interface formation. However, for higher Ag contents beyond this level, the GMR was reduced in line with the structural degradation revealed by XRD and SNMS. For the highest Ag contents (above about 10 at.\%), the GMR exhibited a behavior characteristic of a granular magnetic alloy, in agreement with the results of the structural study.

KEYWORDS: electrodeposition, Co/Cu multilayer, Ag surfactant, GMR, depth profile analysis, XRD

\footnotetext{
${ }^{+}$Corresponding author. E-mail: bakonyi.imre@wigner.mta.hu
} 


\section{Introduction}

One of the major obstacles hindering the realization of a high giant magnetoresistance (GMR) in electrodeposited (ED) ferromagnetic/non-magnetic (FM/NM) multilayers is that the non-magnetic spacer layer cannot be electrodeposited as pinhole-free for layer thicknesses which are required to achieve high GMR and an oscillatory GMR behavior [1].

Sputtered $\mathrm{Co} / \mathrm{Cu}$ multilayers [2-4] exhibit an oscillatory GMR and a room-temperature GMR of about $50 \%$ and $20 \%$ at about 1 and $2 \mathrm{~nm} \mathrm{Cu}$ layer thicknesses, respectively. At these particular spacer thicknesses, a strong antiferromagnetic (AF) exchange coupling exists between the adjacent magnetic layers. It gives rise then to a large GMR effect since the AF coupling ensures an antiparallel alignment of the magnetizations of the neighboring magnetic layers in zero magnetic field. For spacer thicknesses between these so-called AF maxima, the exchange coupling is predominantly ferromagnetic. Thus, due to the parallel alignment of the layer magnetizations even in zero magnetic field, no GMR effect occurs.

By contrast, in ED Co/Cu multilayers [5-9], the GMR shows a monotonous increase with increasing $\mathrm{Cu}$ layer thickness up to about 4 to $5 \mathrm{~nm}$; for thicker $\mathrm{Cu}$ layers, the GMR decreases again. The lack of oscillatory GMR was evidenced as a sign for the absence of a significant AF coupling [8]. At $\mathrm{Cu}$ layer thicknesses below about $2 \mathrm{~nm}$, most studies on $\mathrm{ED} \mathrm{Co} / \mathrm{Cu}$ multilayers [1,8] indicated a complete absence of GMR: an anisotropic magnetoresistance (AMR) $[10,11]$ effect occurred instead. This latter feature hints at the bulk-like behavior of the $\mathrm{ED} \mathrm{Co} / \mathrm{Cu}$ multilayers due to a direct coupling of adjacent magnetic layers via pinholes for small spacer thicknesses.

It is known that even at larger nominal thicknesses for which the spacer layer is continuous, a correlated undulation of the layer planes can lead to a so-called "orange-peel" effect [12] that results in an FM coupling. This effect was also considered to explain the absence of oscillatory GMR in ED $\mathrm{Co} / \mathrm{Cu}$ multilayers by Shima et al. [13]. Another mechanism for interlayer coupling was revealed by the theoretical modeling of Altbir and coworkers [14,15], who have shown that atomic step irregularities at the FM/NM layer interfaces can yield either AF or FM magnetostatic coupling, depending on the relative positions of the step at the interfaces opposite across the spacer.

The above experiences evidence the need for a better control of interfaces, both in terms of interface and layer-plane irregularities on an atomic scale (step defects and intermixing) and on a larger scale (layer-plane undulations). In the case of $\mathrm{Co} / \mathrm{Cu}$ multilayers, it is believed [16] that the problem partly stems from the difference in the in-plane interatomic distances in 
$\mathrm{Cu}$ and $\mathrm{Co}$. Namely, the slightly smaller Co atoms can conveniently form a continuous layer down to a fairly small coverage (small nominal uniform thicknesses) on the surface lattice provided by the slightly larger $\mathrm{Cu}$ atoms as substrate. On the other hand, the larger $\mathrm{Cu}$ atoms cannot coherently form an epitaxial layer over the underlying matrix spanned by a lattice of smaller $\mathrm{Co}$ atoms. Therefore, in lack of other constraints, $\mathrm{Cu}$ atoms on a Co surface tend to form islands (three-dimensional growth) to reduce the lattice strain produced by the lattice misfit and to relax to their equilibrium nearest-neighbor distances as soon as possible. This feature was also revealed earlier for evaporated $\mathrm{Co} / \mathrm{Cu}$ multilayers by Eckl et al. [17] who suggested that, e.g., grain boundaries can constitute such less tightly packed locations on the Co surface where the formation of $\mathrm{Cu}$ nuclei can start.

The imperfect nucleation of $\mathrm{Cu}$ on $\mathrm{Co}$ (weak heteroepitaxy) has also been revealed in attempts to produce $\mathrm{Co} / \mathrm{Cu}$ multilayers with continuous $\mathrm{Cu}$ layers by the molecular-beam epitaxy (MBE) method [18]. It was found that face-centered cubic (fcc) twin formation in the $\mathrm{Cu}$ layers gave rise to a lack of continuity enabling a direct FM coupling between Co layers.

The sputtering technique mainly differs from electrodeposition, evaporation and MBE in the more energetic (highly non-equilibrium), random and high-flux deposition of atoms onto the substrate which does not give a chance for the atoms to diffuse on the surface and to find their equilibrium (lowest-energy) positions. This can be an explanation for the relatively easy formation of very thin ( $1 \mathrm{~nm}$ or less) and still continuous $\mathrm{Cu}$ layers between Co layers in sputtered $\mathrm{Co} / \mathrm{Cu}$ multilayers, which then enable the development of an oscillatory exchange coupling via the NM spacer and the associated oscillatory GMR [2-4]. For other techniques allowing an equilibration of the atoms being deposited, alternative routes are required to achieve a layer-by-layer growth and the formation of better interfaces.

It has been recognized in recent decades that the application of surfactants $[19,20]$ can efficiently promote layer-by-layer growth since some specific adatoms are able to alter the kinetics of deposition and the surface energy of the specimen being grown. In the case of MBE-grown $\mathrm{Co} / \mathrm{Cu}$ multilayers, the use of $\mathrm{Pb}$ as surfactant could be demonstrated to yield continuous $\mathrm{Cu}$ layers down to 3 monolayer coverage [18] and such $\mathrm{Co} / \mathrm{Cu}$ multilayers have, indeed, exhibited a strong AF coupling [21]. In the application of a surfactant element for the growth of a $\mathrm{Co} / \mathrm{Cu}$ multilayer [20], one monolayer of the surfactant atoms is deposited first on the substrate. The surfactant atoms, due to their high mobility and low surface energy, will then climb up on top of the multilayer and build a kind of floating sheet. This capping monolayer of surfactant atoms forces a layer-by-layer growth mode even for $\mathrm{Cu}$ atoms on top of a Co surface. A combined experimental and simulation study [22] has, furthermore, 
revealed the atomistic mechanism by which the surfactant induces a layer-by-layer growth. This proceeds via suppressing the hopping mechanism of surface diffusion over the terraces and promoting atomic exchange between surfactant and deposited atoms, both at the terraces and across the steps.

Further studies [23-27] have also demonstrated the beneficial effect of $\mathrm{Pb}$ as surfactant in evaporated and MBE-grown FM/NM multilayers. Several other surfactant elements such as $\mathrm{Bi}$ [26-30], Ag [31-35], Au [36] and In [37] have been similarly found to induce layer quality improvement for physically deposited multilayers.

Specifically, the use of $\mathrm{Ag}$ as surfactant in the growth of $\mathrm{Co} / \mathrm{Cu}$ multilayers by physical methods resulted in smoother interfaces by inhibiting the interfacial alloying across the interfaces [31-34]. The growth mechanisms induced by $\mathrm{Ag}$ as a surfactant in GMR multilayers were investigated by An et al. [35] by using interface-sensitive X-ray anomalous scattering techniques. These results also suggested that the addition of Ag during deposition suppresses interfacial intermixing. X-ray diffuse scattering profiles showed that the interfacial lateral correlation length of the Ag-doped multilayer is longer than that of the undoped multilayer and does not change significantly after annealing, suggesting that the addition of Ag gives rise to smoother interfaces and results in a good thermal stability [35].

The simultaneous addition of $\mathrm{Ag}$ and $\mathrm{Au}$ to the spacer layer of sputtered $\mathrm{Co}-\mathrm{Fe} / \mathrm{Cu}$ multilayers (as high as 10 to 15 at.\% of $\mathrm{Ag}$ and 5 to 10 at.\% of $\mathrm{Au}$ ) has also resulted in a significant improvement in layer quality $[38,39]$. The reason for this effect may be that these additives modified the spacer layer formation mechanism, leading finally to much smoother multilayers and better GMR behavior. The beneficial effect was explained [38] by the surfactant effect of mainly the Ag atoms having high mobility and always tending to move to the surface whereas Au atoms were rather included in the spacer layer. This explanation was also supported by molecular dynamics simulations [38], which revealed that, whereas for pure $\mathrm{Cu}$ spacer layers, pin-hole formation is quite frequent in the spacer, in $\mathrm{Cu}-\mathrm{Ag}$ - $\mathrm{Au}$ spacers such pin-hole formation did not occur. The presence of these non-magnetic elements in the $\mathrm{Cu}$ spacer as additives is not deleterious for the GMR since the spacer resistivity remains still fairly low for these element combinations.

As far as electrodeposition is concerned, a few attempts [40,41] have only been made for the use of metals as surfactant. Specifically, it was shown that, with the help of $\mathrm{Pb}$ and $\mathrm{Cu}$ as surfactants, Ag growth on Au could be manipulated in a manner so as to obtain smooth layers up to a coverage of 200 monolayers with atomically-flat surfaces. The situation is more complex for ED multilayers and still fully unexplored. In the present paper, we will describe 
the first results for the use of a surface-active additive to the electrochemical bath with the aim of modifying the spacer layer growth in FM/NM multilayers produced by electrodeposition.

Based on the favorable experiences with the use of $\mathrm{Ag}$ as surfactant in physical deposition methods for the growth of $\mathrm{Co} / \mathrm{Cu}$ multilayers, it was decided to investigate the effect of $\mathrm{Ag}$ addition to the spacer layer on the structure and GMR of ED Co/Cu multilayers. After elaborating an appropriate electrolyte formulation for the electrodeposition of the three metals $\mathrm{Co}, \mathrm{Cu}$ and $\mathrm{Ag}$ from a single bath, multilayers with various amounts of $\mathrm{Ag}$ incorporated into the $\mathrm{Cu}$ spacer layer were prepared. The impact of $\mathrm{Ag}$ addition on the multilayer microstructure and on the GMR behavior was investigated by using energy dispersive X-ray spectroscopy (EDX), secondary neutral mass spectroscopy (SNMS), X-ray diffraction (XRD) and magnetoresistance measurements. After presenting the EDX, SNMS, XRD and magnetoresistance results, we will attempt to give a comprehensive discussion of the impact of $\mathrm{Ag}$ incorporation in $\mathrm{ED} \mathrm{Co} / \mathrm{Cu}(\mathrm{Ag})$ multilayers on layer formation and GMR.

\section{Experimental}

Materials and chemicals. - The low solubility of most Ag salts in water imposes a strong limitation on the anion choice for studying the impact of $\mathrm{Ag}$ addition to $\mathrm{ED} \mathrm{Co} / \mathrm{Cu}$ multilayers. Although electrodeposition of silver from $\mathrm{AgNO}_{3}$ solution is possible, the reduction of the nitrate ions makes it impossible to deposit a Co layer. Therefore, perchloratetype solutions can only be used [42]. The Co/Ag system did not need to be studied in detail since it was published prior to the start of the present work [43].

All chemicals used for the solution preparation were of analytical grade. It was found that the addition of boric acid is necessary to obtain a continuous and fairly smooth deposit that can be reliably contacted for the magnetoresistance measurements. The composition of the solution was optimized for the concentrations of $\mathrm{Co}\left(\mathrm{ClO}_{4}\right)_{2}$ and $\mathrm{H}_{3} \mathrm{BO}_{3}$ as specified below. Two stock solutions were used in the experiments. The first one contained $\mathrm{Co}\left(\mathrm{ClO}_{4}\right)_{2}$ (0.2 mol dm${ }^{-3}$; Alfa Aesar), $\mathrm{Cu}\left(\mathrm{ClO}_{4}\right)_{2}\left(0.015 \mathrm{~mol} \mathrm{dm}^{-3}\right.$; Alfa Aesar), and $\mathrm{H}_{3} \mathrm{BO}_{3}(0.2 \mathrm{~mol}$ $\mathrm{dm}^{-3}$; Reanal). The second one contained $\mathrm{AgClO}_{4}$ (Alfa Aesar) instead of $\mathrm{Cu}\left(\mathrm{ClO}_{4}\right)_{2}$ but with the same metal ion concentration, while the other components and their concentrations were the same as in the first electrolyte. An appropriate mixture of the two stock solutions was used for the preparation of $\mathrm{ED} \mathrm{Co} / \mathrm{Cu}(\mathrm{Ag})$ multilayers with various $\mathrm{Ag}$ concentrations in the spacer 
layer. The $\mathrm{Ag}^{+}$ion concentration of the electrolyte solution used for multilayer preparation will be given with the following ratio throughout the entire work: $[\mathrm{Ag}+] /\left(\left[\mathrm{Ag}^{+}\right]+[\mathrm{Cu} 2+]\right)$.

Sample preparation. - Electrodeposition was performed at ambient conditions in a tubular cell with an upward facing horizontal cathode of $1.5 \mathrm{~cm}^{2}$ surface area $[44,45]$. An EF453 type potentiostat/galvanostat (Electroflex, Hungary) served as current source. A saturated calomel electrode (SCE) was used as reference electrode which was connected to the cell via an external vessel filled up with an electrolyte having a composition identical to the bath used for deposition except for the $\mathrm{Ag}$ salt. In the liquid junction, $\mathrm{Ag}^{+}$was not present because it would form $\mathrm{AgCl}$ precipitate in the reference electrode.

The substrate was a metal-coated Si wafer with the (100) orientation. A 5-nm-thick Cr adhesive layer was followed by a 20 -nm-thick $\mathrm{Cu}$ conductive layer; both layers were produced by evaporation. The mean surface roughness of the $\mathrm{Si} / \mathrm{Cr} / \mathrm{Cu}$ substrates determined with atomic force microscopy was found to be between 1 and $3 \mathrm{~nm}$ [46].

A galvanostatic/potentiostatic $(\mathrm{G} / \mathrm{P})$ pulse combination $[1,44,47]$ was used to produce the $\mathrm{Co} / \mathrm{Cu}(\mathrm{Ag})$ multilayers. The magnetic Co layer was deposited at $-19.2 \mathrm{mAcm}^{-2}$ current density in galvanostatic mode. Lower current densities led to fragmented deposits, while at higher current densities the shiny metallic surface turned into a dull one with many nonmetallic black spots. For establishing the optimum potential for depositing the non-magnetic layer, the following procedure was followed.

First, cyclic voltammograms $(\mathrm{CV})$ were recorded at a scan rate of $5 \mathrm{mV} / \mathrm{s}$ in order to reveal the potential ranges of the deposition of the three components $(\mathrm{Co}, \mathrm{Cu}$ and $\mathrm{Ag})$. The CVs are shown in Fig. 1 for the electrolytes with $1 \%$ and $8 \% \mathrm{Ag}^{+}$ion concentrations. It is noted that the $\mathrm{CV}$ of the baths with an $\mathrm{Ag}^{+}$ion concentration below $1 \%$ was very similar to that shown for $1 \% \mathrm{Ag}^{+}$ion concentration, whereas for $15 \%$ it was similar to that shown for $8 \%$. Independently of the $\mathrm{Ag}^{+}$ion concentration, the onset of $\mathrm{Cu}$ and $\mathrm{Co}$ deposition in the cathodic scans was at about $0 \mathrm{~V}$ and $-0.9 \mathrm{~V}$, respectively. The onset of $\mathrm{Ag}$ deposition is at about $+0.3 \mathrm{~V}$ which is clearly visible only for $8 \%$ and $15 \% \mathrm{Ag}^{+}$ion concentrations as shown in the inset of Fig. 1. At lower $\mathrm{Ag}^{+}$ion concentrations, the partial current of $\mathrm{Ag}$ deposition is negligibly small. The anodic stripping peaks with a current maximum at about $-0.1 \mathrm{~V}$ and $+0.4 \mathrm{~V}$ correspond to $\mathrm{Co}$ and $\mathrm{Cu}$ dissolution, respectively. An Ag stripping peak is not visible because the amount of Ag codeposited is mostly very small and hence, its current contribution remains hidden in the wide $\mathrm{Cu}$ dissolution peak. In the potential range, where predominantly 
$\mathrm{Cu}$ deposition can be expected to occur, the limiting current density increases by a factor of 2 upon increasing the $\mathrm{Ag}^{+}$ion concentration from $1 \%$ to $8 \%$. This can certainly be ascribed to the presence of an increased amount of $\mathrm{Ag}^{+}$ions in the bath, although it is hard to assess the actual microscopic mechanism by which the $\mathrm{Ag}^{+}$ions induce this large change.

The electrochemically optimized value for the deposition potential of the non-magnetic layer was determined by measuring the current transients during the non-magnetic layer deposition pulse according to a previously described method [1,47]. This procedure yielded a potential of $-0.585 \mathrm{~V}$ vs. SCE for each $\mathrm{Ag}^{+}$ion concentration used and this value was then applied for the deposition of the non-magnetic layers in the multilayer. The use of this optimized value ensures that neither a dissolution of the previously deposited magnetic layer (Co), nor a codeposition of the magnetic atoms into the non-magnetic layer can occur. The limiting current density of $\mathrm{Cu}$ deposition was found to be about $-0.5 \mathrm{~mA} / \mathrm{cm}^{2}$ for the bath without $\mathrm{Ag}^{+}$ions (Fig. 1). By taking the ratio of the limiting currents and the magnetic layer deposition current, we can estimate the $\mathrm{Cu}$ content in the magnetic layer to be about $2.5 \mathrm{at} \%$, which corresponds to our previous experience with electrodeposited $\mathrm{Co} / \mathrm{Cu}$ multilayers [48]. It can be assumed that the $\mathrm{Cu}$ content in the magnetic layer can also be similar for the other multilayers prepared from baths with not too high $\mathrm{Ag}^{+}$ion concentrations.

In the case of samples prepared for structural and magnetoresistance studies, the nominal thicknesses of the magnetic and non-magnetic layer were $3 \mathrm{~nm}$ and $7 \mathrm{~nm}$, respectively (by assuming a $100 \%$ current efficiency for both kinds of layer). The number of bilayer repetitions was chosen to give a total multilayer thickness of $800 \mathrm{~nm}$.

A different procedure was necessary for the preparation of multilayers intended for the depth profile analysis by SNMS. Once the $\mathrm{Co} / \mathrm{Cu}(\mathrm{Ag})$ multilayer was formed in a manner as described above, a Ni support layer was deposited on top of it to enable us to peel off the deposits from the substrate without any significant damage. The solution for plating the $\mathrm{Ni}$ adlayer was composed of $\mathrm{NiSO}_{4}\left(0.60 \mathrm{~mol} \mathrm{dm}^{-3}\right), \mathrm{Na}_{2} \mathrm{SO}_{4}\left(0.20 \mathrm{~mol} \mathrm{dm}^{-3}\right), \mathrm{MgSO}_{4}(0.16 \mathrm{~mol}$ $\left.\mathrm{dm}^{-3}\right), \mathrm{NaCl}\left(0.12 \mathrm{~mol} \mathrm{dm}{ }^{-3}\right)$ and $\mathrm{H}_{3} \mathrm{BO}_{3}\left(0.40 \mathrm{~mol} \mathrm{dm}^{-3}\right)$. The latter bath was optimized to obtain a Ni supportive layer with a sufficient tensile strength and with a low internal stress on the $\mathrm{Co} / \mathrm{Cu}(\mathrm{Ag})$ multilayers. The preparation of the supporting layer was performed by exchanging the electrolyte but without disassembling the cell. This method assured that the same area was covered with the supporting layer as for the multilayer itself. The minimum total thickness of the supporting layer was about $3 \mu \mathrm{m}$ in order to achieve a sufficient toughness. Further details of the sample preparation process can be found in earlier papers 
[46,49-52]. After the electrochemical sample preparation, the Si wafer was broken behind the multilayer sample in a manner that the deposit itself remained intact. Then, the deposit could easily be peeled off from the $\mathrm{Si}$ wafer so that the separation took place at the $\mathrm{Si} / \mathrm{Cr}$ interface. The surface of the resulting Cr-capped sample was as smooth as the Si wafer, making it particularly appropriate for a depth profile study. In order to achieve a sufficient spatial resolution along the depth [46], the individual layer thicknesses in the $\mathrm{Co} / \mathrm{Cu}(\mathrm{Ag})$ multilayers prepared for the SNMS study were chosen as high as 10 or $15 \mathrm{~nm}$ whereby keeping an equal thickness for the magnetic and non-magnetic layers. The total thickness of the SNMS multilayers was 400 or $450 \mathrm{~nm}$.

Composition analysis - The overall composition of the multilayer films was determined by energy-dispersive X-ray spectroscopy (EDX) in a JEOL JSM 840 scanning electron microscope. The RÖNTEC EDX unit equipped with a Si detector was operated at $25 \mathrm{kV}$. The chemical analysis was carried out for spots of about $1 \mathrm{~mm}^{2}$ surface area each. The composition was measured on at least three different spots for every sample.

Composition depth profile analysis - The composition depth profile analysis of the samples was performed by the SNMS method with an instrument of INA-X type (SPECS GmbH, Berlin, Germany) in the direct bombardment mode by using $\mathrm{Ar}^{+}$ions with a fairly low energy for sputtering $\left(E_{\mathrm{Ar}^{+}}=350 \mathrm{eV}\right)$. The erosion area was confined to a circle of $2 \mathrm{~mm}$ in diameter by means of a Ta mask. The lateral homogeneity of the ion bombardment was checked by a profilometric analysis of the craters sputtered. The calculation methods of the composition vs. depth function was described earlier ([52] and references cited therein).

Structural study - The structure of the $\mathrm{Co} / \mathrm{Cu}(\mathrm{Ag})$ multilayers was investigated by wideangle XRD. The XRD experiments were performed in symmetrical mode on a RD7 diffractometer (FPM/Seifert) working in the conventional Bragg-Brentano geometry [53,54]. The diffractometer was equipped with a sealed X-ray tube with copper anode and with a curved graphite monochromator located in the diffracted beam.

Magnetoresistance measurements - The magnetoresistance (MR) characteristics of the deposits were studied at room temperature. The magnetoresistance was measured with a 4point-in-line configuration up to $H=8 \mathrm{kOe}$ external magnetic field in the field-in-plane, current-in-plane mode. The magnetoresistance was measured both in longitudinal (LMR) and 
transverse (TMR) configurations with the magnetic field and the current parallel or perpendicular to each other, respectively. The MR ratio was defined as $\Delta R / R_{\mathrm{O}}=[R(H)-$ $\left.R_{\mathrm{O}}\right] / R_{\mathrm{O}}$ where $R(H)$ and $R_{\mathrm{O}}$ are the resistance values measured in a magnetic field $H$ and at the resistance peak around zero magnetic field, respectively. A standard Langevin fitting procedure [55] was applied to the measured $M R(H)$ curves in order to separate the ferromagnetic and superparamagnetic (SPM) contributions to the GMR.

\section{Results}

Overall chemical composition analysis. - The impact of $\mathrm{Ag}^{+}$ion concentration in the bath on deposit composition was studied by a gradual replacement of $\mathrm{Cu}^{2+}$ ions with $\mathrm{Ag}^{+}$ions in the electrolyte. The concentrations of the constituent metals in the multilayered deposits are displayed as a function of the ionic ratio $\left[\mathrm{Ag}^{+}\right] /\left(\left[\mathrm{Ag}^{+}\right]+\left[\mathrm{Cu}^{2+}\right]\right)$ in the bath (Fig. 2).

The overall deposit composition data (Fig. 2a) indicate that with increasing $\mathrm{Ag}^{+}$ion concentration in the electrolyte, the $\mathrm{Co}$ and $\mathrm{Cu}$ content in the multilayered deposits changes continuously (the Co content increases and the $\mathrm{Cu}$ content decreases). Figure $2 \mathrm{a}$ also reveals that for low $\mathrm{Ag}^{+}$ion concentrations in the bath (up to about $2 \%$ ), the $\mathrm{Ag}$ content in the deposit is very small $\left(<1-2\right.$ at.\%). For higher $\mathrm{Ag}^{+}$ion concentrations in the bath, a more rapid increase of the codeposition of Ag can be detected by the EDX analysis. It can be inferred from Fig. 2a that the stronger the degree of the codeposition of $\mathrm{Ag}$ in the deposit, the stronger is the change in the $\mathrm{Cu}$ content and the Co content of the deposit and this correlation is in agreement with the corresponding change in the magnitude of the Ag partial current density according to the cyclic voltammograms (Fig. 1). The increase of the Co molar fraction in the deposit with the increase of the $\mathrm{Ag}^{+}$ion concentration in the bath also means that the partial current density associated with $\mathrm{Cu}$ deposition during the high-current pulse decreases. Hence, a collateral effect of the replacement of $\mathrm{Cu}^{2+}$ with $\mathrm{Ag}^{+}$in the electrolyte is an enhanced decrease of the $\mathrm{Cu}$ content in the magnetic layer.

The decrease in the $\mathrm{Cu}$ content of the deposit can be well understood by the replacement of the $\mathrm{Cu}^{2+}$ ions with $\mathrm{Ag}^{+}$ions. However, one should also notice that at the maximum $\mathrm{Ag}^{+}$ ion concentration used, the Co content of the deposit becomes twice as much as it is in the case of the $\mathrm{Ag}^{+}$-free electrolyte. This clearly shows the complexity of the deposition process.

The measured $\mathrm{Ag}$ and $\mathrm{Cu}$ molar fractions in the non-magnetic spacer (Fig. 2b) are in qualitative agreement with the $\mathrm{Ag}$ and $\mathrm{Cu}$ partial current densities in the diffusion-limited range of $\mathrm{Ag}$ and $\mathrm{Cu}$ deposition (see the $\mathrm{CV}$ curves in the inset of Fig. 1). The deposition of 
$\mathrm{Ag}$ at low $\mathrm{Ag}^{+}$ion concentrations in the bath appears slightly hindered with respect to what could be expected from the solution composition (cf. experimental data below the linear reference line $y=x$ in Fig. 2b). Since Ag does not form equilibrium alloys with Co and the mutual solubility of $\mathrm{Ag}$ and $\mathrm{Cu}$ is also very limited, the deposition of $\mathrm{Ag}$ requires a high activation energy due to the hindered nucleation of $\mathrm{Ag}$ crystals on any of the other two metals. Therefore, as long as the concentration of the $\mathrm{Ag}^{+}$ions in the bath is very low, they remain fairly inactive in the deposition process itself, although the standard potential of the $\mathrm{Ag}^{+} / \mathrm{Ag}$ system is by far more positive than that of the other metal ions/metal pairs present in the system. At sufficiently high $\mathrm{Ag}^{+}$ion concentrations, however, the $\mathrm{Ag}$ deposition becomes a dominant process as indicated by the fact that the Ag molar fraction in the deposit increases to a much higher value than the ionic ratio in the solution. The mechanism for this different behavior may be that once the $\mathrm{Ag}$ content in the deposit reaches a critical value, the subsequently incoming $\mathrm{Ag}$ atoms can be more easily accommodated in the surface. Once this nucleation barrier is overcome, the electrochemical nobility of $\mathrm{Ag}$ ensures the excessive $\mathrm{Ag}$ deposition upon a further increase of the $\mathrm{Ag}^{+}$ion concentration in the bath.

Composition depth profile analysis by SNMS. — In order to get information on the layer formation in the investigated multilayers, a composition depth profile study was performed by the SNMS technique. For a clear observation of the individual layers, the layer thicknesses had to be set somewhat higher than those favored in the magnetoresistance study. Based on our earlier work [46], the layer thicknesses were chosen to be 10 or $15 \mathrm{~nm}$ (and being equal for $\mathrm{Co}$ and $\mathrm{Cu}$ in each SNMS sample). The results of the composition depth profile analysis by SNMS are presented in Fig. 3.

The SNMS depth profile curves for the Ag-free multilayer are shown in Fig. 3a. The general features, which are similar also for the other two samples, are as follows. The sputtering procedure starts with the Cr layer (Fig. 3a). The high molar fraction of $\mathrm{Cr}$ at the beginning of the sputtering process indicates that there was no reaction between the Cr buffer layer and the Si substrate (from which the native oxide was not removed prior to the evaporation process). This was already pointed out by the successful separation of the Si substrate from the Cr buffer and the easy peeling off of the metallic coating from the substrate without damaging the multilayer structure. Then a thick $\mathrm{Cu}$ layer follows which is comprised of the 20-nm-thick substrate layer and the first electrodeposited $\mathrm{Cu}$ layer $(15 \mathrm{~nm})$. The composition oscillation caused by the multilayer structure composed of 15-nm-thick Co and $\mathrm{Cu}$ layers can be seen thereafter with decreasing oscillation amplitude. Since the multilayer 
was covered with a $\mathrm{Ni}$ supporting layer, the transition is indicated by vanishing $\mathrm{Co}$ and $\mathrm{Cu}$ signals and a rising Ni signal whereby the Ni molar fraction tends to approach 1 afterwards. The thickness of this interface is characteristic of the surface roughness of the sample.

For the $\mathrm{Ag}$-free $\mathrm{Co} / \mathrm{Cu}$ multilayer (Fig. 3a), as many as 10 concentration waves can be clearly observed up to a depth of $300 \mathrm{~nm}$ for both $\mathrm{Co}$ and $\mathrm{Cu}$. The average distance between the neighboring Co maxima is $30.0 \mathrm{~nm}$; i.e., exactly the value designed during the sample preparation process. This finding validates the assumption of a $100 \%$ current efficiency. The envelope curve of the decay of the composition oscillation for depths beyond $300 \mathrm{~nm}$ is mainly determined by the development of the surface roughness of the sample [46]. At high depth, where the planar sputtering front crosses the tilted layer interfaces randomly, a smearing out of the concentration profile can be observed. However, it must be emphasized that the lack of concentration waves at high depths cannot be taken as evidence of the disappearance of the periodic composition modulation (i.e., a well-defined layered structure) but rather it stems from the surface roughening for larger total multilayer thickness.

When the bath contained a minor amount of $\mathrm{Ag}^{+}$ions (see Fig. $3 \mathrm{~b}$ for a sample with $2.7 \% \mathrm{Ag}^{+}$ion concentration in the bath and with $10 \mathrm{~nm}$ layer thicknesses), the overall $\mathrm{Co}$ content of the deposit remained the same as without the Ag addition (see Fig. 3a). In this sense, the SNMS measurement supports the results of the EDX analysis that indicated a minor change only in the Co content for low $\mathrm{Ag}^{+}$ion concentrations. The difference with respect to the Ag-free sample occurs in the first $\mathrm{Cu}$ wave in the SNMS depth profile. This wide peak was split up into two peaks, and the average of the centers of the two $\mathrm{Cu}$ peaks coincides with the first peak of the Ag signal in the spectrum. This can be explained with the preferential deposition of the noblest $\mathrm{Ag}$ from the solution at the beginning of the electrodeposition process when neither of the reactants was yet depleted in the vicinity of the cathode. Since the relative $\mathrm{Ag}^{+}$content of the solution was small, the initially deposited $\mathrm{Ag}$ was covered with $\mathrm{Cu}$, which gives rise to the second $\mathrm{Cu}$ peak as the $\mathrm{Ag}$ yield decreases after the $\mathrm{Ag}$ deposition reaches the diffusion-limited regime.

For this multilayer prepared with $2.7 \% \mathrm{Ag}^{+}$ion concentration in the bath (Fig. 3b), the SNMS spectra showed a composition oscillation in the first $100 \mathrm{~nm}$ only. This value is barely the third of the depth until concentration oscillations could be seen in the absence of Ag (Fig. 3a). This fact indicates that the incorporation of $\mathrm{Ag}$ leads to a faster increase of the surface roughness with multilayer thickness than it was in the absence of Ag, or a small amount of incorporated Ag results in a loss of the ordered structure of the sample by some other means. 
The oscillation period observed was $19.9 \mathrm{~nm}$ that agrees again very well with the designed value $(20.0 \mathrm{~nm})$. As for the Ag-free sample, the disappearance of the composition modulation is probably the consequence of an increased surface roughness. A comparison of Figs. 3a and $3 \mathrm{~b}$ shows that the roughness reaches a critical level at smaller total multilayer thickness than for the Ag-free sample.

Based on the concentration oscillation period, a comparison of the Ag molar fraction of the deposit with that of the two other components of the sample reveals where the Ag can be found in the layered structure. The inset in Fig. $3 \mathrm{~b}$ presents the $\mathrm{Ag}$ and $\mathrm{Cu}$ molar fractions at such magnifications as to better visualize the periodicity of the signals. This comparison shows that the Ag incorporation takes place mostly in the non-magnetic layer. The curves did not indicate any $\mathrm{Ag}$ accumulation at either of the $\mathrm{Co} / \mathrm{Cu}(\mathrm{Ag})$ and $\mathrm{Cu}(\mathrm{Ag}) / \mathrm{Co}$ interfaces of the $\mathrm{Cu}$ layer in the multilayer structure since the $\mathrm{Ag}$ and $\mathrm{Cu}$ concentration oscillations are completely in phase.

The average $\mathrm{Ag}$ content of the sample prepared with $2.7 \% \mathrm{Ag}^{+}$ion concentration in the solution was 1.8 at.\% Ag from the EDX analysis (see Fig. 2a), and the SNMS results in Fig. $3 \mathrm{~b}$ indicate a comparable $\mathrm{Ag}$ content.

The SNMS depth profile results for a multilayer prepared with an $\mathrm{Ag}^{+}$ion concentration of $8.0 \%$ in the bath are shown in Fig. 3c. A concentration oscillation with very weak amplitude can be observed in the SNMS profile only in the close vicinity of the substrate. According to Fig. 2a, the $\mathrm{Ag}$ content in this multilayer is already as high as about 8 at.\% and this agrees fairly well with the height of the Ag hump at low depths (ca. $70 \mathrm{~nm}$ ). The SNMS profiles in Fig. 3c reveal that in this $\mathrm{Ag}^{+}$ion concentration range, the sample is characterized by a deterioration and, finally, a loss of the layered structure in the course of the deposition. The unexpected increase in the Co molar fraction at $d>100 \mathrm{~nm}$ depths indicates that the high Co content found in the bulk composition analysis at high silver concentrations occurs after the deposition of a certain amount of metal whose thickness is small as compared to the total sample thickness of the multilayers analyzed. For this sample, which was prepared from a solution of $8 \% \mathrm{Ag}^{+}$, the multilayer/Ni cover layer transition range was much wider than for the other samples with low or zero Ag content. This broad transition is due to the very strong surface roughening as a consequence of the incorporation of a high amount of $\mathrm{Ag}$ into the deposit. 
Structural study by XRD. - Segments of the XRD patterns recorded for the multilayers with $\mathrm{Ag}^{+}$ion concentrations of $0,0.5 \%$, and $1.2 \%$ in the bath near the main $(111) \mathrm{fcc}-\mathrm{Co} / \mathrm{Cu}$ multilayer reflection are shown in Fig. 4. With reference to Fig. 2b, the Ag content in the nonmagnetic layer of the multilayers prepared with $0.5 \%$ and $1.2 \% \mathrm{Ag}^{+}$ion concentration is 0.15 at. $\%$ and 0.36 at. $\%$, respectively.

In addition to the experimental data, Fig. 4 also displays the results of the fitting of the (111) multilayer peak and the superlattice satellites by symmetrical Pearson VII functions [56]. The presence of the superlattice satellites in the XRD patterns confirms the result of SNMS depth profiling regarding the multilayer structure. The weak intensity of the superlattice satellites endorses the degradation of the multilayer structure that was already indicated by SNMS depth profiles.

From the positions of the neighboring superlattice satellites, the bilayer thicknesses were determined by the expression [57]

$$
\Lambda_{X R D}=\frac{\lambda}{2\left(\sin \theta_{n+1}-\sin \theta_{n}\right)} .
$$

The bilayer thicknesses were found to be between 10.3 and $10.5 \mathrm{~nm}$ for all three multilayers, which values correspond well to the nominal bilayer thickness $\Lambda_{\text {nom }}=10 \mathrm{~nm}$. The bilayer thickness determined from the $\mathrm{XRD}$ data $\left(\Lambda_{\mathrm{XRD}}\right)$ is slightly larger than $\Lambda_{\mathrm{nom}}$, as it is usually observed for $\mathrm{ED} \mathrm{Co} / \mathrm{Cu}$ multilayers [9]. In Eq. (1), the positions of two neighboring satellites are denoted as $\theta_{\mathrm{n}}$ and $\theta_{\mathrm{n}+1}$, whereas $\lambda$ is the wavelength of the X-rays.

From the position of the main peaks (satellites with $n=0$, which are labelled as ML in Fig. 4), the mean interplanar spacings $\langle d\rangle$ for the lattice planes (111) were calculated by using the expression

$$
\langle d\rangle=\frac{\lambda}{2 \sin \theta_{0}}
$$

In the sample without $\mathrm{Ag}$, a mean interplanar spacing of $0.2073 \mathrm{~nm}$ was determined for the lattice planes (111). This interplanar spacing agrees very well with the mean interplanar spacing $0.2075 \mathrm{~nm}$ calculated by the expression [58]

$$
\langle d\rangle=\frac{d_{C o} t_{C o}+d_{C u} t_{C u}}{t_{C o}+t_{C u}}
$$

for a $\mathrm{Co} / \mathrm{Cu}$ multilayer with the nominal thicknesses $t_{\mathrm{Co}}=3 \mathrm{~nm}$ and $t_{\mathrm{Cu}}=7 \mathrm{~nm}$. The distances of the lattice planes (111) for the pure constituent metals, $d_{\mathrm{Co}}=0.2046 \mathrm{~nm}$ and $d_{\mathrm{Cu}}=0.2087$ $\mathrm{nm}$, were calculated from the lattice parameters $0.35443 \mathrm{~nm}$ for fcc-Co [59] and $0.361505 \mathrm{~nm}$ 
for fcc- $\mathrm{Cu}$ [60]. In the multilayers deposited with $\mathrm{Ag}$ surfactant, a slight increase of the interplanar spacing was observed. However, this increase was much smaller than it should be for Ag fully incorporated in the crystal structure of $\mathrm{Cu}$, which is the main component of the periodic non-magnetic layers.

In the samples prepared from a bath containing $0 \%$ and $0.5 \% \mathrm{Ag}^{+}$ion concentration, distinct XRD lines from pure $\mathrm{Cu}$ and $\mathrm{Co}$ (dashed lines in Fig. 4) could be identified by the fitting in addition to the main multilayer peak and the weak superlattice satellites. These XRD lines are narrower than the satellites. This indicates the formation of continuous $\mathrm{Cu}$ and $\mathrm{Co}$ domains, the thickness of which is larger than the thickness of the periodic part of the multilayer. However, we should keep in mind that because of the very weak integral intensity of the additional peaks, these pure metal phases constitute a very minor phase only, their total volume in the sample being at most $1 \%$ or even less. The features of the pure metal diffraction lines suggest that these phases form narrow columns along the growth direction, i.e., perpendicular to the layer planes. The integral intensity of these XRD lines decreases with increasing $\mathrm{Ag}$ content in the multilayers. For the multilayer prepared from a bath with $1.2 \%$ $\mathrm{Ag}^{+}$ion concentration, these XRD lines disappear. Therefore, the codeposition of a small amount of silver with copper seems to reduce the formation of large continuous regions consisting of cobalt or copper, which latter are not parts of the multilayer stack.

According to the XRD results shown in Fig. 5, the incorporation of a very large amount of Ag to the deposit leads to a drastic deterioration of the multilayer structure as already indicated by SNMS. Moreover, for the multilayer prepared from a bath with $15 \% \mathrm{Ag}^{+}$ion concentration (with reference to Fig. 2a, this multilayer contained nearly as much $\mathrm{Ag}$ as $\mathrm{Cu}$ ), a segregation of silver from copper could be concluded from the XRD pattern. The double peak at $2 \theta \approx 38.5^{\circ}$ indicates a non-uniform distribution of the impurities in Ag. The left-hand peak corresponds to pure silver, the right-hand one to a mixture of $\mathrm{Ag}$ and probably $\mathrm{Cu}$.

Magnetoresistance characteristics. - All investigated $\mathrm{Co} / \mathrm{Cu}(\mathrm{Ag})$ multilayers exhibited a GMR effect in that both the longitudinal and transverse components of the magnetoresistance were negative, with a slight difference in the LMR and TMR magnitudes due to the inherent AMR effect within the ferromagnetic regions. Figure 6 displays the measured $M R(H)$ curves for the four multilayers subjected to the above described XRD study, the results of which were presented in the previous section. These $M R(H)$ curves represent the typical magnetoresistance behavior of all other samples. 
The $M R(H)$ curves in Fig. 6a show saturation in fairly low magnetic fields (at about 2 kOe) and this indicates that the observed GMR can be ascribed to spin-dependent scattering events for electrons in a fairly well-defined layered structure consisting of an alternating sequence of ferromagnetic layers and non-magnetic spacer layers [1,55]. Such a behavior cannot be a direct consequence of the absence of $\mathrm{Ag}^{+}$ions in the electrolyte since other multilayers prepared similarly from a silver-free electrolyte exhibited rather the behavior shown in Figs. 6b and 6c where the $M R(H)$ curves have a slightly non-saturating character up to $8 \mathrm{kOe}$. The non-saturating component can be ascribed to the presence of SPM regions in the magnetic layers [1,55]. This feature was the most typical behavior of the $M R(H)$ curves of multilayers investigated in the present work. In such cases, the experimental $M R(H)$ curves were analyzed by a standard procedure [55] in order to separate the FM and SPM contributions to the GMR $\left(\mathrm{GMR}_{\mathrm{FM}}\right.$ and $\mathrm{GMR}_{\mathrm{SPM}}$, respectively). In this standard procedure, it is assumed that above a certain saturation field $H_{\mathrm{s}}$, the field dependence of the magnetoresistance $M R(H)$ could be described by a Langevin function $L(\mu H / k T)$ :

$$
M R(H)=M R_{\mathrm{FM}}+G M R_{\mathrm{SPM}} \cdot L(\mu H / k T),
$$

whereby $M R_{\mathrm{FM}}=A M R+G M R_{\mathrm{FM}}$ is a constant for $H>H_{\mathrm{s}}$. The $\mathrm{GMR}_{\mathrm{FM}}$ contribution stems from spin-dependent scattering events for electron paths between two FM regions, whereas the $\mathrm{GMR}_{\mathrm{SPM}}$ term from spin-dependent scattering events for electron paths between a FM and a SPM region.

For the $M R(H)$ curves shown in Figs. 6b and 6c, the SPM component is relatively small with respect to the FM component. On the other hand, for the $M R(H)$ curves shown in Fig. 6d, not only the magnitude of the total observed GMR is much smaller than for the other three multilayers, but also the non-saturating component, i.e., the SPM contribution clearly dominates the observed GMR.

These features will be demonstrated quantitatively in the following by presenting the decomposed $M R(H)$ curves for cases with small and large SPM contributions to the GMR. Figure 7 displays the results of decomposition analysis for the LMR component of the measured $M R(H)$ curves for two samples shown in Figs. 6c and 6d (the TMR data show the same qualitative behavior). Together with the experimental data, the decomposed FM and SPM contributions are plotted for these two $\mathrm{Co} / \mathrm{Cu}(\mathrm{Ag})$ multilayers. The decomposition reveals that for low Ag content in the non-magnetic spacer (0.36 at.\%, Fig. 7a), the FM contribution dominates whereas for very high $\mathrm{Ag}$ content (44.7 at.\%, Fig. 7b), the SPM 
contribution dominates the observed GMR. A further support for the different magnetic characteristics of these two multilayers is provided by a comparison of their low-field hysteresis behavior. For the sample with dominant $\mathrm{GMR}_{\mathrm{FM}}$ contribution (Fig. 7a), the peak positions of the $M R(H)$ curves correspond well to the coercivity values measured for ED $\mathrm{Co} / \mathrm{Cu}$ multilayers with similarly dominant $\mathrm{GMR}_{\mathrm{FM}}$ term [8]. On the other hand, the almost complete absence of coercivity (or hysteresis) in the $M R(H)$ curves shown in Fig. $7 \mathrm{~b}$ indicates a dominant SPM behavior.

By performing the decomposition procedure for all the multilayers, the saturation components of the $\mathrm{GMR}_{\mathrm{FM}}$ and $\mathrm{GMR}_{\mathrm{SPM}}$ contributions have been determined. The sum of these two saturation values gives the total saturation value $\mathrm{GMR}_{\mathrm{s}}($ total) for each sample. Since the difference between the longitudinal and transverse values was typically small, for simplicity, we have averaged these total values for a given sample. The average $<\mathrm{GMR}_{\mathrm{S}}$ (total) $>$ data are displayed in Fig. 8 as a function of the $\mathrm{Ag}^{+}$ion concentration in the bath. The samples were grouped into several series according to their preparation batches for the following reason. Samples prepared in the same batch (i.e., on substrates cut from the same metallized Si wafer) usually exhibited a consistent behavior. On the other hand, for samples prepared in various series with similar variation of the $\mathrm{Ag}^{+}$ion concentration in the bath, the absolute value of the GMR was not always reproducible even if the same trend was obtained for the GMR evolution. According to our previous experience with the GMR of ED $\mathrm{Co} / \mathrm{Cu}$ multilayers, this uncertainty may have been most probably due to an occasional improper batch of the $\mathrm{Si} / \mathrm{Cr} / \mathrm{Cu}$ substrate. Unfortunately, it is not feasible to assess the surface quality for each substrate before deposition. On the other hand, due to the high sensitivity of the GMR effect to microstructural features of the deposited multilayer films, substrate quality differences can easily show up in the observed GMR magnitudes which values sometimes differ by a factor of two for nominally identical multilayers.

By taking into account this uncertainty factor, the GMR data in Fig. 8 can be best evaluated as follows. The multilayers deposited from the silver-free bath exhibited a total $\mathrm{GMR}_{\mathrm{S}}$ value as high as about 8 to $10 \%$. Actually, this is rather surprising since for comparable Co layer thicknesses, the $\mathrm{GMR}_{\mathrm{S}}$ value of $\mathrm{ED} \mathrm{Co} / \mathrm{Cu}$ multilayers [8] reaches such high values around 4 to $5 \mathrm{~nm}$ spacer thickness and for $7 \mathrm{~nm}$ spacer thickness, most studies indicated already a reduced $\mathrm{GMR}_{\mathrm{S}}$ value. It appears as if the perchlorate bath itself had a beneficial effect on layer formation, at least as far as the GMR magnitude is concerned since the high GMR values were not accompanied by clear XRD multilayer satellites. 
According to Fig. 8, in the two series labeled as (1-4) and (51-55), the addition of a small amount of $\mathrm{Ag}^{+}$ions to the bath resulted definitely in an improvement of the GMR magnitude. For further additions of $\mathrm{Ag}^{+}$ions to the bath (typically with concentrations above $1 \%$ ), the GMR magnitude showed an overall decrease down to very small GMR values for the highest $\mathrm{Ag}^{+}$ion concentrations applied.

Figure 8 also displays the $\mathrm{GMR}_{\mathrm{SPM}}$ data (filled squares) for all the multilayers. Apart from the last two samples (11 and $15 \% \mathrm{Ag}^{+}$), the SPM contribution is generally much smaller than the $\mathrm{GMR}_{\mathrm{FM}}$ term, i.e., these multilayers exhibit a relatively well-defined layered structure with a dominating multilayer-type GMR. For the two outstanding samples, which have already a very high $\mathrm{Ag}$ content in the spacer layer (37 and 45 at.\%, respectively), the SPM term is comparable or even almost equal to the FM term. This means that the magnetic layer is definitely fragmented and a large fraction of the fragments exhibits a SPM behavior. This situation corresponds well to the conclusions deduced above from both the SNMS and XRD studies for large Ag contents in the spacer layers.

As one possible origin of an initial increase of the GMR for small $\mathrm{Ag}^{+}$ion concentrations in the bath, we have to consider also the chemical analysis results presented in Fig. $2 \mathrm{~b}$. In the $\mathrm{Ag}^{+}$ion concentration range (below about $1 \%$ ) where an improvement of GMR could be inferred in Fig. 8, the Ag content in the non-magnetic layer is very small, definitely below about 1 at.\%. Therefore, the observed initial GMR increase can be the result of the incorporation of the small amount of $\mathrm{Ag}$ atoms in the non-magnetic spacer layer. Another explanation may be that the presence of $\mathrm{Ag}^{+}$ions in the electrolyte affects the growth mode of the multilayer which, on the other hand, exerts an influence on GMR via microstructural modifications.

\section{Discussion}

$\mathrm{Co} / \mathrm{Cu}(\mathrm{Ag})$ multilayers with low amount of $\mathrm{Ag}$ incorporated. - According to the SNMS results, multilayers prepared at low $\mathrm{Ag}^{+}$ion concentration in the bath exhibit a well-defined layered structure in the near-substrate region. Although multilayers for SNMS studies were prepared with larger individual layer thicknesses than the multilayers for XRD studies and for GMR measurements, we can assume that the key sample features are the same also for the smaller bilayer thicknesses. At the same time, the rapid observed smearing of the SNMS composition modulation along the growth direction also revealed that incorporating about 2 at. $\% \mathrm{Ag}$ in the multilayer led already to some degradation of the layered structure. This could 
have been the consequence of fluctuations of the individual layer thicknesses or of the bilayer thickness with the progress of growth.

The XRD revealed superlattice satellites around the main fcc(111) multilayer peak. This confirmed the result of SNMS regarding the presence of a layered structure near the substrate and its corrugation towards the sample surface. The weak and broad superlattice reflections $[57,58]$ elucidated the origin of the corrugations, which is mainly the variation of the bilayer thickness in the multilayer stack. According to Eq. (1), the variation of the bilayer thickness leads to a change of the satellite positions, while the main multilayer peak does not shift until the thickness ratio of the individual layers in the bilayer remains unchanged, cf. equations (2) and (3). Thus the variation of the bilayer thickness, which is accompanied by the increase of the interface roughness, blurs the superlattice satellites, by making them broader and their maximum intensity weaker $[53,54,57,58]$. Especially in multilayers with a large bilayer thickness (nominally $10 \mathrm{~nm}$ in the present case), where the distances between neighboring superlattice satellites are small, broadened satellites overlap mutually and apparently merge with the main multilayer peak.

Still, it was shown above that all ED $\mathrm{Co} / \mathrm{Cu}(\mathrm{Ag})$ multilayers investigated in the present study exhibited a clear GMR effect. Up to 8 at.\% Ag incorporated in the multilayers, the observed GMR could be mostly ascribed to a dominating $\mathrm{GMR}_{\mathrm{FM}}$ term, whereas the GMR $_{\text {SPM }}$ term was found to be much smaller. The fact that the observed GMR is dominated by the $\mathrm{GMR}_{\mathrm{FM}}$ term can be best interpreted by assuming that these samples consist of layered ferromagnetic regions separated by layered non-magnetic regions. Only electrons travelling from one FM region to an adjacent one, which is separated by a non-magnetic spacer from the first FM region and has a differently oriented magnetization, can undergo the kind of spindependent scattering events which yield a multilayer-type GMR behavior (dominant $\mathrm{GMR}_{\mathrm{FM}}$ term) as observed. At the large spacer layer thickness of $7 \mathrm{~nm}$, probably no antiferromagnetic coupling exists between the magnetic layers [8] and the observed GMR arises due to the random alignment of the magnetizations of adjacent layers [8,61]. In spite of the relatively large bilayer thickness $(\Lambda=10 \mathrm{~nm})$, these $\mathrm{Co} / \mathrm{Cu}(\mathrm{Ag})$ multilayers prepared from a perchlorate bath exhibited a GMR effect at least as large as commonly observed in ED $\mathrm{Co} / \mathrm{Cu}$ multilayers obtained from sulfate-type baths with smaller bilayer repeats [5-9,53,54,62,63].

Thus, the SNMS, XRD and magnetoresistance results are all compatible with the conclusion that the present $\mathrm{ED} \mathrm{Co} / \mathrm{Cu}(\mathrm{Ag})$ multilayers below about 8 at.\% $\mathrm{Ag}$ incorporated exhibit a multilayer structure even if this layered structure is far from perfect. The 
imperfections stem mainly from the lateral fluctuations of the bilayer thickness, which increase gradually the interface roughness during the multilayer growth and lead to a corrugation of the layer planes. Furthermore, a small fraction of the magnetic layers may exhibit SPM behavior and these regions are magnetically decoupled from the dominating FM sections of the magnetic layers. Apparently, a fairly small amount of Ag (less than about 1 at.\%) incorporated in the multilayer induces slight changes in the layer structure and/or in the morphology of the interfaces that results in a clear increase in the GMR magnitude. One of the consequences of the addition of the $\mathrm{Ag}$ surfactant is the suppression of the $\mathrm{Cu}$ and $\mathrm{Co}$ precipitates outside of the multilayer structure. At Ag contents beyond this level, the lattice distortion effects of the Ag atoms in the spacer dominate and this leads then to a reduction of the GMR.

It should also be noted additionally that according to the work of Kubota et al. [4] on sputtered $\mathrm{Co} / \mathrm{Cu}$ multilayers, a large GMR effect and an oscillatory GMR behavior with the concomitant alternating AF/FM coupling could be demonstrated for samples which hardly exhibited superlattice satellite reflections although the observed magnetotransport behavior could only arise from a fairly well defined layered structure. This implies that a large GMR and its oscillatory behavior can occur even in the absence of a perfect periodic superlattice. Of course, the absence of well-observable satellites is an indication for a non-perfect multilayer structure but not for the complete absence of a layered structure as discussed above.

$\mathrm{Co} / \mathrm{Cu}(\mathrm{Ag})$ multilayers with high amount of $\mathrm{Ag}$ incorporated. - At 8 at.\% $\mathrm{Ag}$ in the multilayer, the degree of the superstructure deterioration reached a level, which made nearly impossible to resolve the periodic composition modulations by SNMS even in the nearsubstrate region, probably due to the rapidly increasing surface roughness. Owing to the limited miscibility of $\mathrm{Cu}$ and $\mathrm{Ag}$, such a high $\mathrm{Ag}$ content in the multilayer (which corresponds to more than 10 at.\% $\mathrm{Ag}$ in the spacer layer) leads to the formation of $\mathrm{Ag}$ domains, which may grow as dendrites and thus increase the actual cathode surface (via increased roughening). Such crystalline Ag domains were detected by XRD at the highest Ag content incorporated (roughly when half of the $\mathrm{Cu}$ atoms were replaced by Ag atoms). Surprisingly, some fraction of the deposit even at this high $\mathrm{Ag}$ content contained bits of a superlattice structure, which provided very weak superlattice satellites in the XRD pattern (Fig. 5). However, the magnetoresistance measured in two samples with the highest amount of $\mathrm{Ag}$ incorporated (above 20 at.\% Ag) revealed that the SPM contribution to the GMR was already 
comparable or equal to the FM contribution. This is a clear indication that these samples are strongly disrupted and that they exhibit largely a granular-type structure.

\section{Summary}

In the present study, the influence of $\mathrm{Ag}$ incorporation into $\mathrm{ED} \mathrm{Co} / \mathrm{Cu}$ multilayer stacks on the structure and GMR was investigated. The aim was to find out if a small amount of $\mathrm{Ag}$ in the non-magnetic spacer layer has a similar effect on layer formation and, thus, on GMR as reported for physically deposited $\mathrm{Co} / \mathrm{Cu}$ multilayers, where $\mathrm{Ag}$ was found to act as an appropriate surfactant element with beneficial effects.

After elaborating an appropriate electrolyte formulation for the electrodeposition of the three metals $\mathrm{Co}, \mathrm{Cu}$ and $\mathrm{Ag}$ from a single perchlorate bath, multilayers were prepared with different amounts of $\mathrm{Ag}^{+}$ion concentration in the electrolytic bath. The incorporation of $\mathrm{Ag}$ in the multilayers was fairly modest (1 at.\% $\mathrm{Ag}$ or less) for $\mathrm{Ag}^{+}$ion concentrations up to about $2 \%$, but increased much more strongly for further additions of $\mathrm{Ag}^{+}$ions.

A clear GMR effect was observed in all multilayers investigated. Although a slight SPM contribution to the GMR was present in almost all samples, the observed GMR was mostly dominated by the FM contribution. Such a multilayer-type GMR behavior is due to spindependent scattering events of electrons travelling between two ferromagnetic regions with non-aligned magnetizations. This implies that the samples exhibit a layered structure, apart from the highest concentrations of codeposited $\mathrm{Ag}$.

A regular composition modulation was detected by SNMS both without Ag and for low Ag contents in the spacer (less than about 2 at.\% Ag). However, SNMS also revealed that the incorporation of a higher amount Ag definitely caused a deterioration of the layered structure.

Very faint satellite reflections were only present in the XRD patterns. The low satellite intensity could be ascribed to significant bilayer thickness fluctuations. We could see differences in the XRD patterns for samples with different Ag contents which indicate microstructural changes but these changes could not be unambigously assigned to the observed changes in the GMR.

According to the magnetoresistance data, the GMR magnitude increased initially for small $\mathrm{Ag}^{+}$ion concentrations in the bath (corresponding to $\mathrm{Ag}$ contents up to at most 1 at.\% in the spacer layer). This implies that a small amount of $\mathrm{Ag}$ has a beneficial effect through slight microstructural modifications. This may occur via an influence of the presence of solvated $\mathrm{Ag}^{+}$ions on nucleation and layer growth on the one hand. Another possibility is that 
$\mathrm{Ag}$ goes to the $\mathrm{Co} / \mathrm{Cu}$ interfaces, where it helps to reduce the lattice misfit between $\mathrm{Cu}$ and $\mathrm{Co}$ (as expected for a surfactant).

It is of special interest that for zero or low Ag contents, the GMR for the $\mathrm{Co}(3 \mathrm{~nm}) / \mathrm{Cu}(7 \mathrm{~nm})$ multilayers was as high as $10 \%$ whereas in previous reports the GMR for 7 $\mathrm{nm}$ spacer thickness was already typically much smaller. A possible origin of this observation can be the use of the perchlorate chemical in the electrochemical bath.

For larger Ag contents, the GMR magnitude gradually decreased and from about 20 at.\% Ag incorporated in the multilayer, the GMR became dominated by an SPM contribution. This indicates a degradation of the multilayer structure that was revealed also by the SNMS analysis results towards higher Ag contents. In agreement with these observations, an XRD study of the sample with the highest Ag content demonstrated the appearance of a separate $\mathrm{Ag}$ phase. The unfavorable mutual nucleation properties of Co and Ag on each other have finally lead to a fragmentation of the magnetic layer as well. This explains the appearance of SPM regions at high $\mathrm{Ag}$ contents which could be inferred from the GMR study.

\section{Acknowledgement}

This work was supported by the Hungarian Scientific Research Fund (OTKA) through Grant \# K 75008. 


\section{References}

1. I. Bakonyi and L. Péter, Progr. Mater. Sci. 55, 107 (2010)

2. D.H. Mosca, F. Petroff, A. Fert, P.A. Schroeder, W.P. Pratt Jr. and R. Laloee, J. Magn.. Magn. Mater. 94, L1 (1991)

3. S.S.P. Parkin, R. Bhadra and K.P. Roche, Phys. Rev. Lett. 66, 2152 (1991)

4. H. Kubota, S. Ishio, T. Miyazaki and Z.M. Stadnik, J. Magn. Magn. Mater. 129, 383 (1994)

5. S.K.J. Lenczowski, C. Schönenberger, M.A.M. Gijs and W.J.M. de Jonge, J. Magn. Magn. Mater. 148, 455 (1995)

6. Q.X. Liu, L. Péter, J. Pádár and I. Bakonyi, J. Electrochem. Soc. 152, C316 (2005)

7. P. Chowdhury, S.K. Ghosh, A. Dogra, G.K. Dey, Y.G. Gowda, S.K. Gupta, G. Ravikumar, A.K. Grover and A.K. Suri, Phys. Rev. B 77, 134441 (2008)

8. I. Bakonyi, E. Simon, B.G. Tóth, L. Péter and L.F. Kiss, Phys. Rev. B 79, 174421 (2009)

9. N. Rajasekaran, L. Pogány, Á. Révész, B.G. Tóth, S. Mohan, L. Péter and I. Bakonyi, J. Electrochem. Soc. 161, D339 (2014)

10. R.M. Bozorth, Ferromagnetism (Van Nostrand, New York, 1951)

11. T.R. McGuire and R.I. Potter, IEEE Trans. Magn. 11, 1018 (1975)

12. H.D. Chopra, D.X. Yang, P.J. Chen, D.C. Parks, and W.F. Egelhoff, Jr., Phys. Rev. B 61, $9642(2000)$

13. M. Shima, L.G. Salamanca-Riba, R.D. McMichael and T.P. Moffat, J. Electrochem. Soc. 148, C518 (2001)

14. D. Altbir, M. Kiwi, R. Ramírez, and I.K. Schuller, J. Magn. Magn. Mater. 149, L246 (1995)

15. P. Vargas and D. Altbir, Phys. Rev. B 62, 6337 (2000)

16. Q.X. Liu, L. Péter, J. Tóth, L.F. Kiss, Á . Cziráki, and I. Bakonyi, J. Magn. Magn. Mater. 280, 60 (2004)

17. Th. Eckl, G. Reiss, H. Brückl and H. Hoffmann, J. Appl. Phys. 75, 362 (1994)

18. J. Camarero, L. Spendeler, G. Schmidt, K. Heinz, J.J. de Miguel and R. Miranda, Phys. Rev. Lett. 73, 2448 (1994)

19. E. Bertel and N. Memmel, App. Phys. A, 63, 523 (1996)

20. J.J. de Miguel and R. Miranda, J. Phys.: Cond. Matter 14, R1063 (2002)

21. J.Camarero, T. Graf, J.J. de Miguel, R. Miranda, W. Kuch, M. Zharnikov, A. Dittschar, C.M. Schneider and J. Kirschner, Phys. Rev. Lett. 76, 4428 (1996)

22. J. Camarero, J. Ferrón, V. Cros, L. Gómez, A.L. Vázquez de Parga, J.M. Gallego, J.E. Prieto, J.J. de Miguel and R. Miranda, Phys. Rev. Lett. 81, 850 (1998)

23. W. F. Egelhoff, P. J. Chen, C. J. Powell, M. D. Stiles, R. D. Mcmichael, C. L. Lin, J. M. Sivertsen, J. H. Judy, K. Takano and A. E. Berkowitz, J. Appl. Phys. 80, 5183 (1996)

24. J. Ferron, L. Gomez, J. M. Gallego, J. Camarero, J. E. Prieto, V. Cros, A. De Parga, J. J. De Miguel, R. Miranda, Surf. Sci. 459, 135 (2000)

25. L. Gómez and J. Ferrón, Phys. Rev. B 64, 033409 (2001)

26. M. Marszałek, A. Polit, V. Tokman, Y. Zabila and I. Protsenko, Surf. Sci. 601, 4454 (2007)

27. A. Polit, M. Kac, M. Krupinski, B. Samul, Y. Zabila and M. Marszalek, Acta Phys. Pol. A 112, 1281 (2007)

28. M. Kamiko, H. Chihaya, W. Sugimoto, J.-H. Xu, I. Kojima and R. Yamamoto, J. Magn. Magn. Mater. 310, 2265 (2007)

29. M. Kamiko, A. Nakamura, K. Aotani and R. Yamamoto, Appl. Surf. Sci. 256, 1257 (2009)

30. M. Kamiko, H. Chihaya, W. Sugimoto, R. Yamamoto, S. Oh, J. Xu and I. Kojima, Surf. Rev. Lett. 13, 201 (2006)

31. S. M. Amir, M. Gupta and A. Gupta, J. Alloy. Comp. 522, 9 (2012) 
32. B. L. Brennan, L. Peterson, R. L. White and B. M. Clemens, Physica B 336, 157 (2003)

33. D. X. Yang, B. Shashishekar, H. D. Chopra, P.J. Chen and W.F. Egelhoff, J. App. Phys. 89, $7121(2001)$

34. H.D. Chopra, D.X. Yang, P.J. Chen and W.F. Egelhoff, Jr., Phys. Rev. B 65, 094433 (2002)

35. Yukai An, Hongdi Zhang, Bo Dai, Zhenhong Mai, Jianwang Cai and Zhonghua Wu, J. Appl. Phys. 100, 023516 (2006)

36. W. F. Egelhoff, P. J. Chen, C. J. Powell, M. D. Stiles, R. D. McMichael, C. L. Lin, J. M. Sivertsen, J. H. Judy, K. Takano and A. E. Berkowitz, J. Appl. Phys. 80, 5183 (1996)

37. W. F. Egelhoff, P. J. Chen, C. J. Powell, M. D. Stiles and R. D. McMichael, J. Appl. Phys. 79, 2491 (1996)

38. W. Zou, H.N.G. Wadley, X.W. Zhou, R.A. Johnson and D. Brownell, Phys. Rev. B 64, $174418(2001)$

39. D. Rafaja, J. Ebert, G. Miehe, N. Martz, M. Knapp, B. Stahl, M. Ghafari, H. Hahn, H. Fuess, P. Schmollngruber, P. Farber and H. Siegle, Thin Solid Films 460, 256 (2004)

40. K. Sieradzki, S.R. Brankovic and N. Dimitrov, Science 284, 138 (1999)

41. S.R. Brankovic, N. Dimitrov and K. Sieradzki, Electrochem. Solid-State Lett. 2, 443 (1999)

42. E. Gomez, J. Garcia-Torres, and E. Valles, Anal. Chim. Acta 602, 187 (2007)

43. J. García-Torres, L. Péter, Á. Révész, L. Pogány and I. Bakonyi, Thin Solid Films 517, 6081-6090 (2009)

44. V. Weihnacht, L. Péter, J. Tóth, J. Pádár, Zs. Kerner, C.M. Schneider and I. Bakonyi, J. Electrochem. Soc. 150, C507 (2003)

45. L. Péter, J. Pádár, E. Tóth-Kádár, Á. Cziráki, P. Sóki, L. Pogány and I. Bakonyi, Electrochim. Acta 52, 3813 (2007)

46. A. Bartók, A. Csik, K. Vad, G. Molnár, E. Tóth-Kádár and L. Péter, J. Electrochem. Soc. 156, D253 (2009)

47. L. Péter, Q.X. Liu, Zs. Kerner and I. Bakonyi, Electrochim. Acta 49, 1513 (2004)

48. I. Bakonyi, L. Péter, Z.E. Horváth, J. Pádár, L. Pogány and G. Molnár, J. Electrochem. Soc. 155, D688 (2008)

49. K. Neuróhr, A. Csik, K. Vad, A. Bartók, G. Molnár and L. Péter, J. Solid State Electrochem. 15, 2523-2544 (2011)

50. A. Csik, K. Vad, E. Tóth-Kádár and L. Péter, Electrochem. Commun. 11, 1289 (2009)

51. L. Péter, A. Csik, K. Vad, E. Tóth-Kádár, Á. Pekker and G. Molnár, Electrochim. Acta 55, 4734 (2010)

52. L. Péter, G. L. Katona, Z. Berényi, K. Vad, G. A. Langer, E. Tóth-Kádár, J. Pádár, L. Pogány and I. Bakonyi, Electrochim. Acta 53, 837 (2007)

53. D. Rafaja, C. Schimpf, V. Klemm, G. Schreiber and I. Bakonyi, L. Péter, Acta Mater. 57, $3211(2009)$

54. D. Rafaja, C. Schimpf, T. Schucknecht, V. Klemm, L. Péter and I. Bakonyi, Acta Mater. 59, 2992 (2011)

55. I. Bakonyi, L. Péter, Z. Rolik, K. Kiss-Szabó, Z. Kupay, J. Tóth, L.F. Kiss and J. Pádár, Phys. Rev. B 70, 054427 (2004)

56. M.M. Hall Jr. V.G. Veerarghavan, H. Rubin and P.G. Winchell, J. Appl. Cryst. 10, 66 (1977)

57. E.E. Fullerton, I.K. Schuller, H. Vanderstraeten and Y. Bruynserade, Physical Review B 45, 9292 (1992)

58. C. Michaelsen, Philos. Mag. A 72, 813 (1995)

59. A. Taylor and R.W. Floyd, Acta Cryst. 3, 285 (1950)

60. H.M. Otte, J. Appl. Phys. 32, 1536 (1961)

61. K. Szász and I. I. Bakonyi, J. Spintr. Magn. Nanomater. 1, 157 (2012)

62. Á. Cziráki, L. Péter, V. Weihnacht, J. Tóth, E. Simon, J. Pádár, L. Pogány, C.M. Schneider, T. Gemming, K. Wetzig, G. Tichy and I. Bakonyi, J. Nanosci. Nanotechnol., 6, 2000 (2006)

63. N. Rajasekaran, J. Mani, B.G. Tóth, G. Molnár, S. Mohan, L. Péter and I. Bakonyi, J. Electrochem. Soc. 162, D204 (2015) 


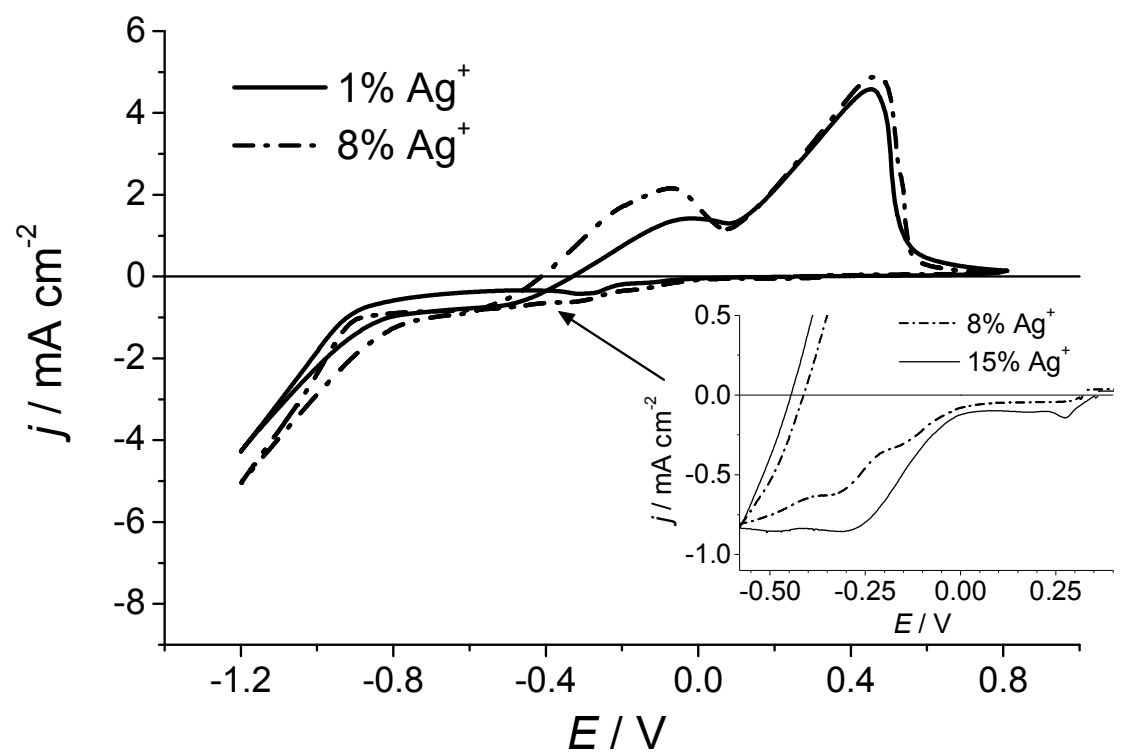

Fig. 1 Cyclic voltammograms of perchlorate electrolytes used for multilayer deposition with $1 \%$ and $8 \% \mathrm{Ag}^{+}$ion concentration as indicated. Inset: Enlarged section of the cyclic voltammograms for the electrolytes with $8 \%$ and $15 \% \mathrm{Ag}^{+}$ion concentration to better reveal the Ag deposition in the potential range from $-0.6 \mathrm{~V}$ to $+0.4 \mathrm{~V}$. 

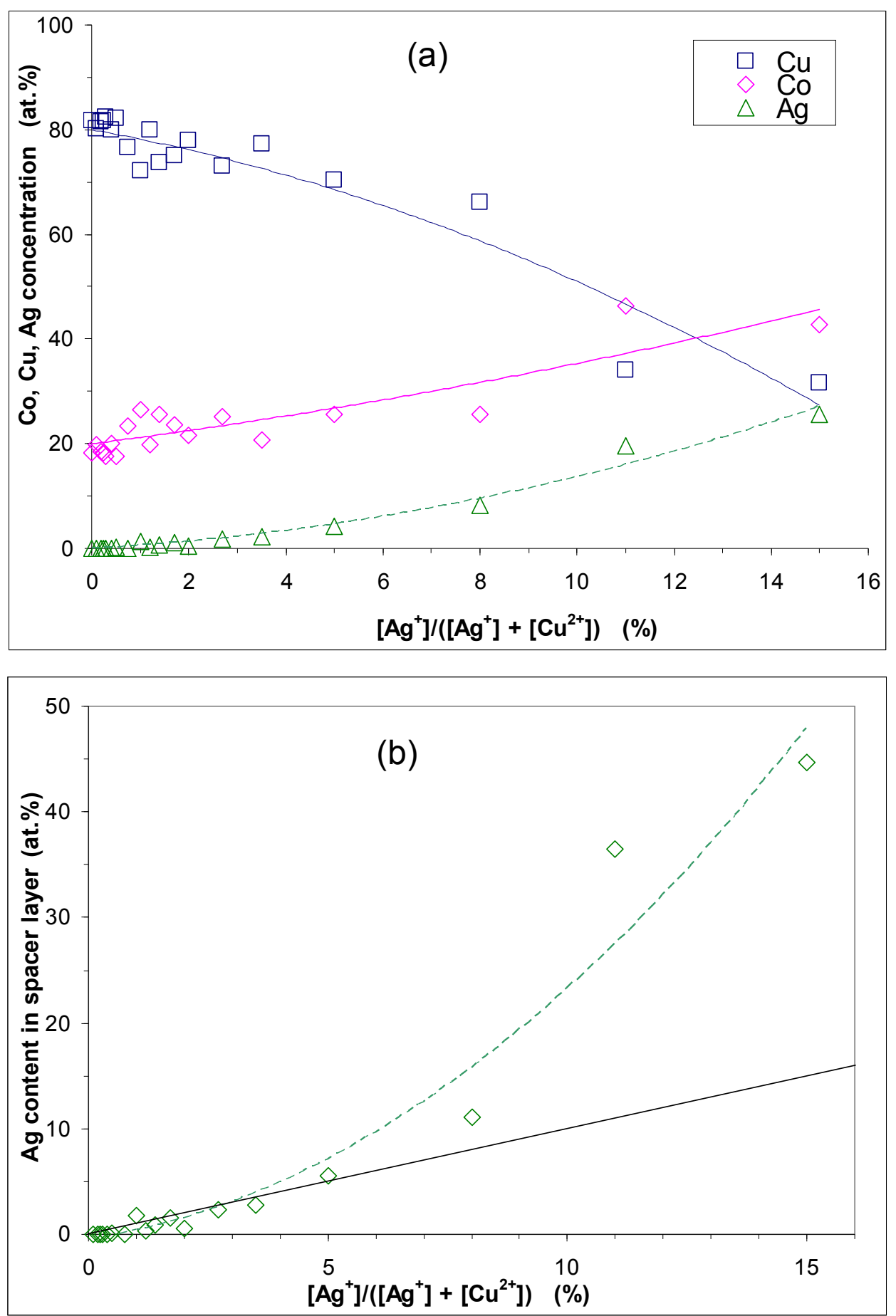

Fig. 2 Composition of the $\mathrm{Co} / \mathrm{Cu}(\mathrm{Ag})$ multilayer deposits as a function of the ionic ratio of the non-magnetic elements in the solution: (a) Molar fraction (in at.\% units) of $\mathrm{Co}, \mathrm{Cu}$ and Ag in the multilayer deposits. The lines indicate only the trend of the concentration variation for each component; (b) Relative molar fraction of $\mathrm{Ag}$ (in at.\% units) with respect to $\mathrm{Cu}$ in the non-magnetic layer of the deposit (data were obtained by assuming here that the amount of $\mathrm{Cu}$ and $\mathrm{Ag}$ in the magnetic layer is negligible). The solid line is the reference line $y=x$ and the dashed line is a guide for the eye only through the analysis results. 

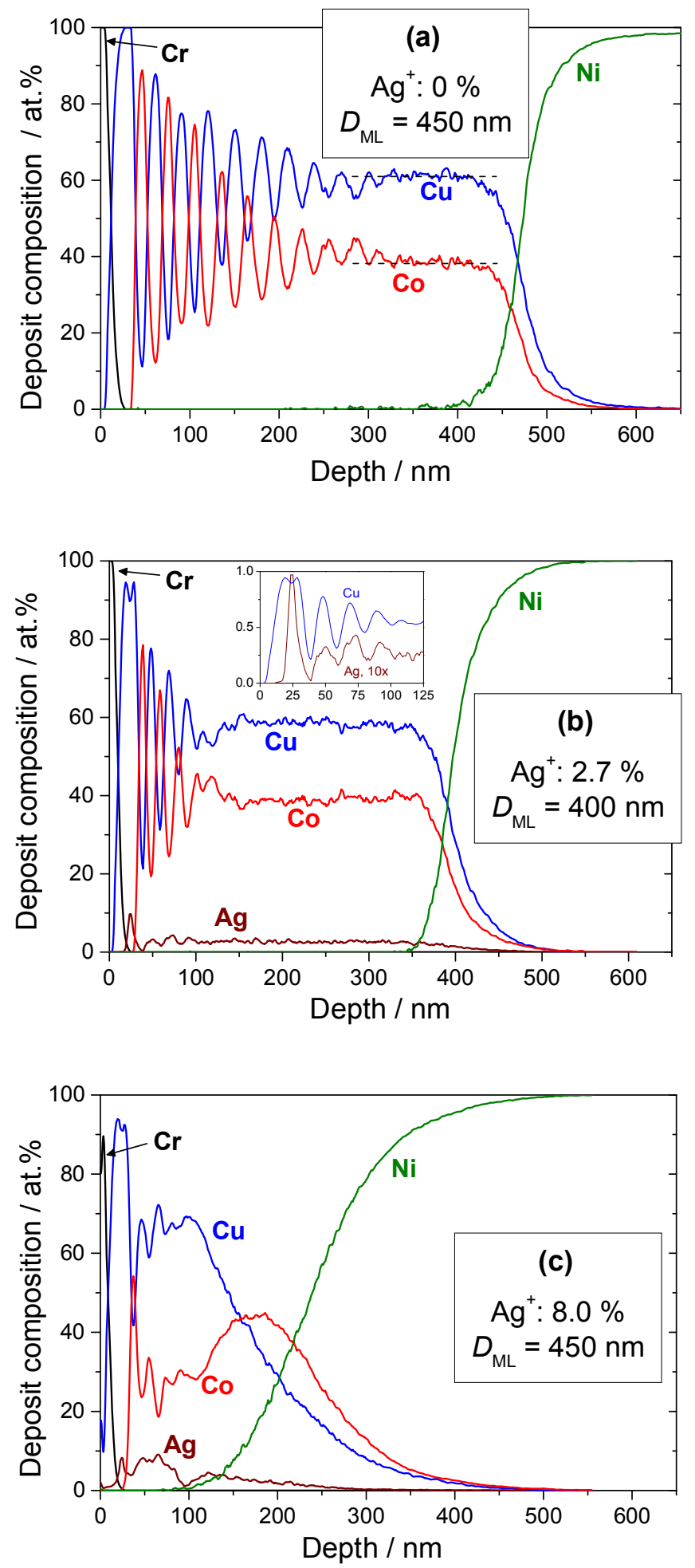

Fig. 3 SNMS depth profiles recorded for Co/Cu(Ag) multilayer samples deposited from solutions with various $\mathrm{Ag}^{+}$ion concentrations in the bath: (a) $d_{\mathrm{Co}}=d_{\mathrm{Cu}}=15 \mathrm{~nm}$; no $\mathrm{Ag}^{+}$ present; (b) $d_{\mathrm{Co}}=d_{\mathrm{Cu}}=10 \mathrm{~nm} ; \mathrm{Ag}^{+}: 2.7 \%$; (c) $d_{\mathrm{Co}}=d_{\mathrm{Cu}}=15 \mathrm{~nm} ; \mathrm{Ag}^{+}: 8.0 \%$. The $\mathrm{Ag}^{+}$ion concentration of the solution given is defined as in Fig. 1. The notation $D_{\mathrm{ML}}$ refers to the total nominal multilayer thickness. 


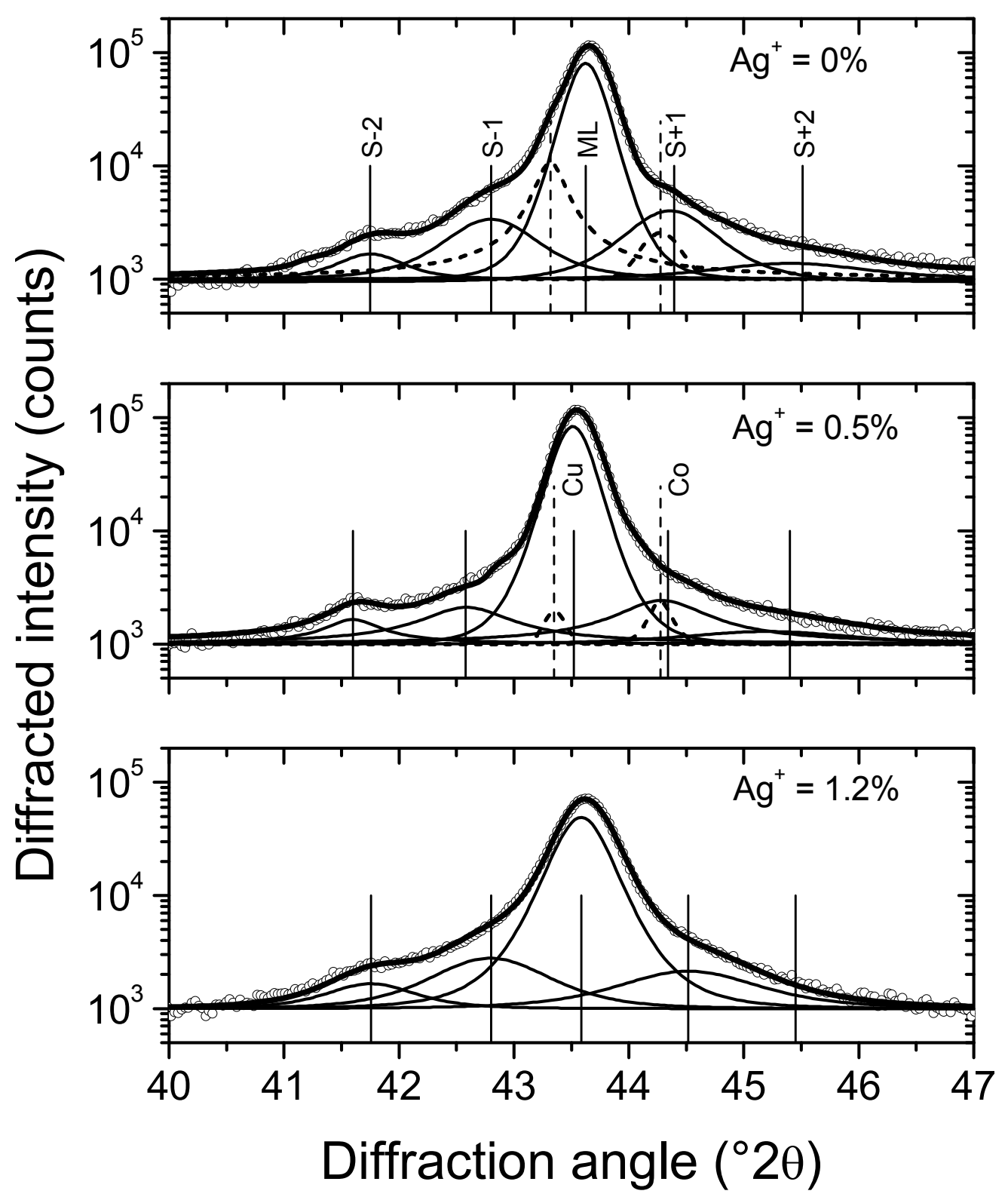

Fig. 4 Sections of the XRD patterns of three ED Co/Cu multilayers taken in the vicinity of the (111) diffraction lines of $\mathrm{fcc}-\mathrm{Co}$ and $\mathrm{fcc}-\mathrm{Cu}$. Top panel: no $\mathrm{Ag}^{+}$ions in the bath; middle panel: $0.5 \% \mathrm{Ag}^{+}$ion concentration in the bath; bottom panel: $1.2 \% \mathrm{Ag}^{+}$ion concentration in the bath. The measured intensities are plotted by open circles, the sum of the fitted intensities by thick solid lines and the intensities of the superlattice satellites by thin solid lines. The dashed lines in the top and middle panels indicate the positions of the diffraction lines from bulk $\mathrm{Cu}\left(\right.$ at $43.32^{\circ} 2 \theta$ ) and bulk Co (at $44.22^{\circ} 2 \theta$ ) regions as deduced from the fitting. The $\Lambda_{X R D}$ values determined by using Eq. (1) are (10.5 \pm 1.0$) \mathrm{nm}$ (top panel), (10.5 \pm 0.2$) \mathrm{nm}$ (middle panel) and $(10.3 \pm 0.2) \mathrm{nm}$ (bottom panel). 


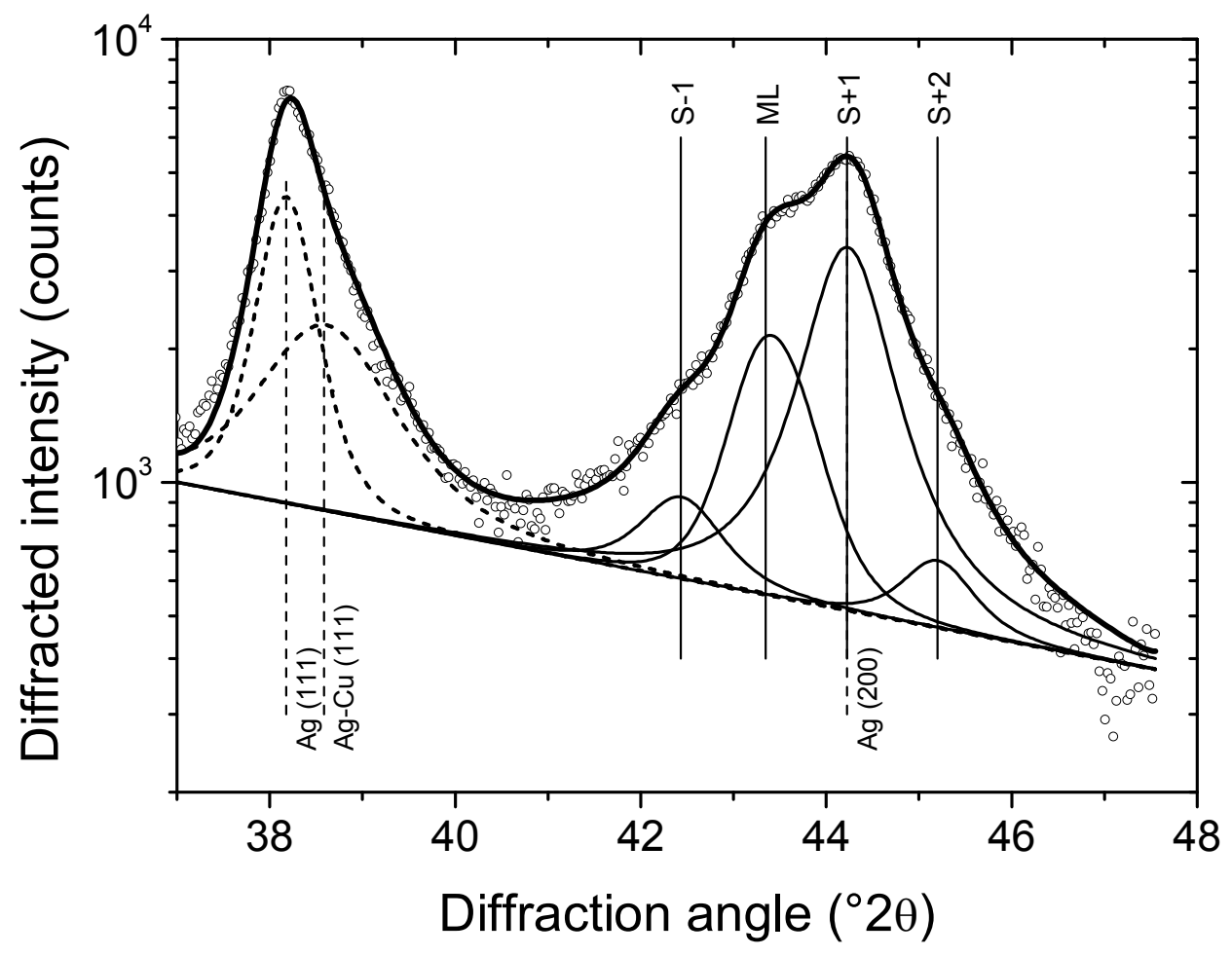

Fig. 5: Low-angle part of the XRD pattern of the Co/Cu(Ag) sample prepared from a bath containing $15 \% \mathrm{Ag}^{+}$ion concentration (according to Fig. $2 a$, the Ag content in this deposit was about 25 at.\%). The measured intensities are plotted by open circles, the sum of the fitted intensities by thick solid lines and the intensities of the main multilayer peak and the superlattice satellites by thin solid lines (the satellite positions yield a $\Lambda_{X R D}$ value of (10.3 \pm 0.2) $\mathrm{nm}$ ). The diffraction lines from $\mathrm{Ag}$ and $\mathrm{Ag}-\mathrm{Cu}$ are plotted by dashed lines. The superlattice satellite $S+1$ is hidden in the diffraction line (200) from Ag. 

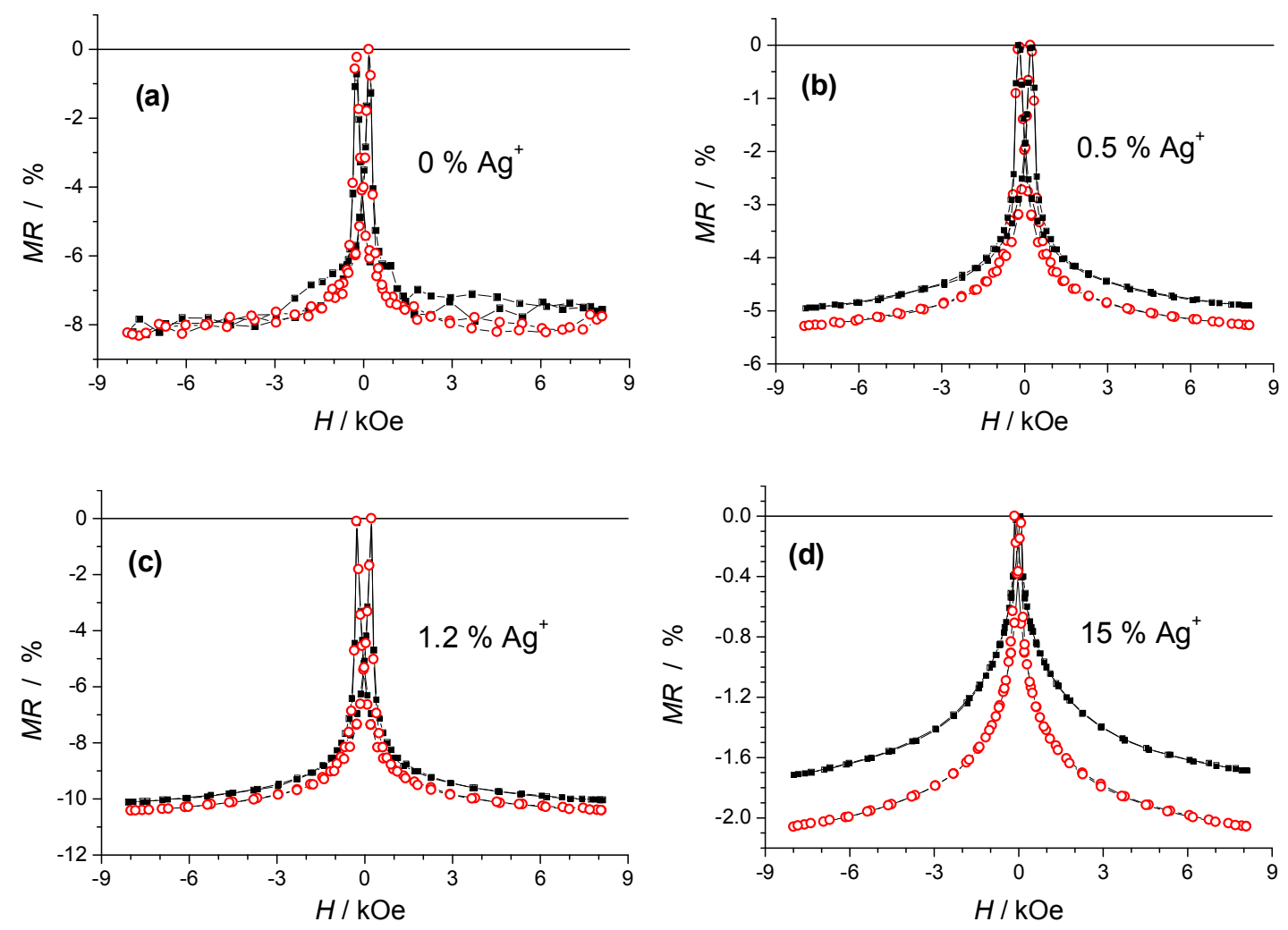

Fig. $6 \mathrm{MR}(H)$ curves measured for the four multilayers investigated by XRD (see Fig. 4), which were prepared with various $\mathrm{Ag}^{+}$ion concentrations in the bath as indicated. Full symbols: LMR component; empty symbols: TMR component. 

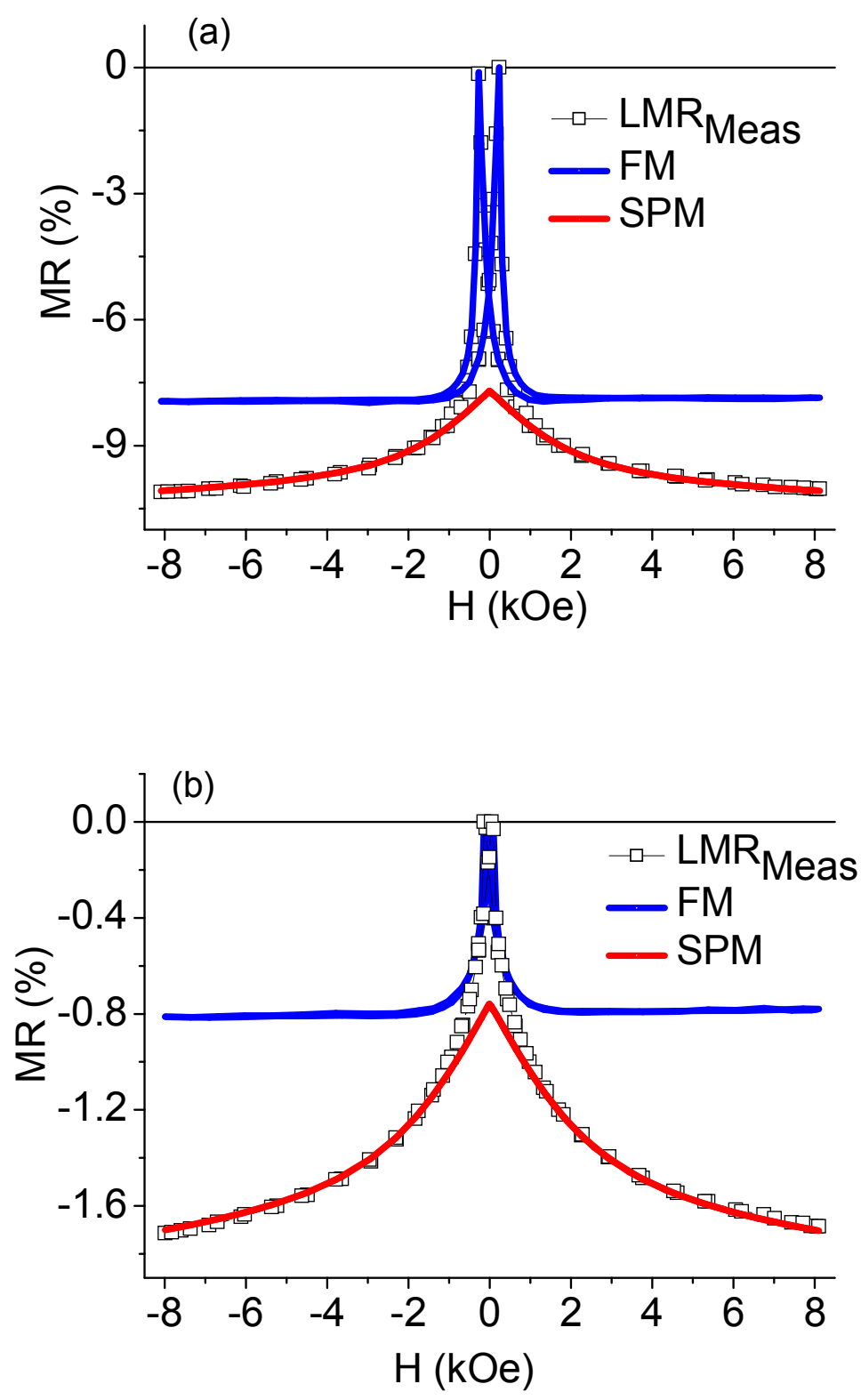

Fig. 7 Decomposition of the measured longitudinal MR component (LMR $\mathrm{Meas}_{\text {) }}$ into ferromagnetic (FM) and superparamagnetic (SPM) contributions for two Co/Cu(Ag) multilayers electrodeposited with (a) $1.2 \% \mathrm{Ag}^{+}$and (b) $15 \% \mathrm{Ag}^{+}$ion concentrations in the bath (the analyzed Ag contents in the non-magnetic layers were 0.36 at.\% and 44.7 at.\%, repspectively). The two samples selected for MR decomposition are those for which the $M R(H)$ curves were shown in Fig. 6c and Fig. 6d, respectively. 


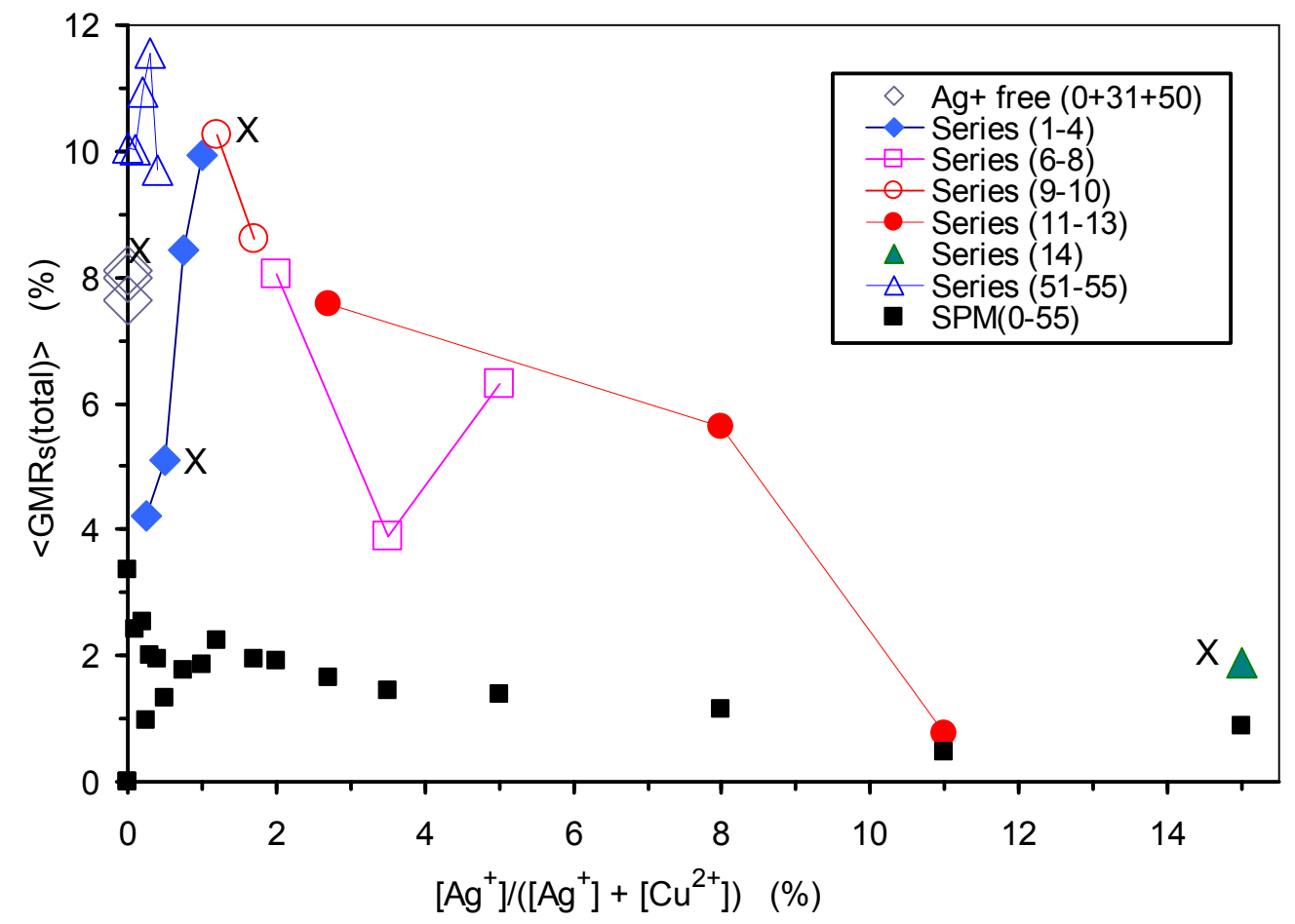

Fig. 8 Total saturation GMR as a function of the relative $\mathrm{Ag}^{+}$concentration in the solution. The averaging indicated by $<>$ refers to an average of the longitudinal and transverse $M R$ components. The data points are grouped in series with samples prepared in the same run (data points connected by thin lines) and the sample serial numbers, which refer to the sequence of sample preparation within the series, are given in the brackets in the legend. The three diamond symbols refer to individual multilayers prepared without $\mathrm{Ag}^{+}$ion addition to the bath at various times during this study. The filled squares show the average saturation SPM component for all the samples. A letter $\mathrm{X}$ was attached to the data points of samples investigated by XRD. 